\title{
Synthesis of some novel spiro heterocycles- Part II
}

\author{
Venkatapuram Padmavathi,* Boggu Jagan Mohan Reddy, Akula Baliah, \\ Adivireddy Padmaja, and Dandu Bhaskar Reddy \\ Department of Chemistry, Sri Venkateswara University, Tirupati-517 502, India \\ E-mail: vkpuram2001@yahoo.com
}

\begin{abstract}
A new class of spiro-pyrimidinones, pyrazolidinones and isoxazolidinones are prepared from 4cyano-4-ethoxycarbonyl-piperidines / tetrahydropyrans / tetrahydrothiopyrans.
\end{abstract}

Keywords: Spiro-pyrimidinones, spiro-pyrazolidinones, spiro-isoxazolidinones, cyclocondensation

\section{Introduction}

During the last one and half decades we have been actively involved in the synthesis of spiro heterocycles. In fact, a variety of compounds viz, spiro-pyrimidinetriones, pyrazolidinediones and isoxazolidinediones have been reported by exploiting the synthetic utility of bis chalcones and bis (styryl) sulfones via double Michael addition followed by reaction with different nucleophiles. ${ }^{1-6}$ In continuation of our sustained interest in this field, new, versatile and multifunctional reactive intermediates 3,3-disubstituted 1,5-diaryl-1,5-pentanediones have been reported. These compounds were used as synthons to get a variety of spiro-heterocycles. ${ }^{7,8}$ Furthermore, the active methylene group present in these compounds was subjected to Knoevenagel condensation with araldehydes under PTC conditions. ${ }^{9}$ Incorporation of heteroatoms in these 3,3-disubstituted 2,4-diaroyl-1,5-diaryl-1,4-pentanedienes resulted 4,4disubstituted 3,5-diaroyl-2,6-diaryl-piperidines / pyrans / thiopyrans. ${ }^{9}$ It is well documented that pyrazole, isoxazole, pyrimidine and thioxopyrimidine derivatives were prepared by treating gemdicarboxylates with hydrazine hydrate, hydroxylamine hydrochloride, urea and thiourea. ${ }^{10-12}$ Adopting the same methodology, dimethyl 3,5-diaroyl-2,6-diaryl- piperidine / tetrahydropyran / tetrahydrothiopyran-4,4-dicarboxylates were used as synthons for spiro-pyrimidinetriones, thioxopyrimidinediones, pyrazolidinediones and isoxazolidinediones. ${ }^{13}$ Encouraged by these results herein we report a new and novel class of spiro heterocycles from 3,5-diaroyl-2,6-diaryl4cyano-4-ethoxycarbonyl- piperidines / tetrahydropyrans / tetrahydrothiopyrans. 


\section{Results and Discussion}

The cyclocondensation of 3,5-diaroyl-2,6-diaryl-4-cyano-4-ethoxycarbonyl- piperidines $\mathbf{1}$, 3,5diaroyl-2,6-diaryl-4-cyano-4-ethoxycarbonyl-tetrahydropyrans $\quad$ 2, 3,5-diaroyl-2,6-diaryl-4cyano4-ethoxycarbonyl-tetrahydrothiopyrans 3 with urea in the presence of NaOMe gave 5amino7,11-diaroyl-8,10-diaryl-3-hydroxy-2,4,9-triazaspiro[5.5] undeca-2,4-dien-1-ones 4, 5amino7,11-diaroyl-8,10-diaryl-3-hydroxy-9-oxa-2,4-diazaspiro [5.5]undeca-2,4-dien-1-ones $\mathbf{5}$ and 5-amino-7,11-diaroyl-8,10-diaryl-3-hydroxy-9-thia-2,4-diazaspiro[5.5]undeca-2,4-dien-1ones 6.

Similar reaction with N,N'-dimethyl urea and thiourea afforded 7,11-diaroyl-8,10-diaryl2,4dimethyl-5-imino-2,4,9-triaza-spiro[5.5] undecane-1,3-diones 7, 7,11-diaroyl-8,10-diaryl2,4dimethyl-5-imino-9-oxa-2,4-diazaspiro[5.5] undecane-1,3-diones 8, 7,11-diaroyl-8,10-diaryl2,4dimethyl-5-imino-9-thia-2,4-diazaspiro[5.5] undecane-1,3-diones $\mathbf{9}$ and 5-amino-7,11diaroyl8,10-diaryl-3-mercapto-2,4,9-triazaspiro[5.5] undeca-2,4-dien-1-ones $\mathbf{1 0}$ 5-amino-7,11diaroyl8,10-diaryl-3-mercapto-9-oxa-2,4-diazaspiro[5.5] undeca-2,4-dien-1-ones 11, 5-amino-7,11diaroyl8,10-diaryl-3-mercapto-9-thia-2,4-diazaspiro[5.5]undeca-2,4-dien-1-ones 12, respectively. Likewise, five membered spiro heterocycles, 4-amino-6,10-diaroyl-7,9-diaryl2,3,8-triazaspiro[4.5]deca-3-en-1-ones 13, 4amino-6,10-diaroyl-7,9-diaryl-8-oxa-2,3diazaspiro[4.5]deca-3-en-1-ones $\quad \mathbf{1 4}$, 4-amino-6,10-diaroyl-7,9diaryl-8-thia-2,3diazaspiro[4.5]deca-3-en-1-ones 15 and 4-amino-6,10-diaroyl-7,9-diaryl-2-oxa3,8diazaspiro[4.5]deca-3-en-1-ones 16, 4-amino-6,10-diaroyl-7,9-diaryl-2,8-dioxa-3azaspiro[4.5]deca-3-en1-ones 17, 4-amino-6,10-diaroyl-7,9-diaryl-2-oxa-8-thia-3-azaspiro[4.5]deca-3-en-1-ones 18 were prepared by refluxing 1/2/3 with hydrazine hydrate and hydroxylamine hydrochloride, respectively (Scheme). The IR spectra of 4-6, 10-18 displayed an absorption band in the region $3200-3330 \mathrm{~cm}^{-1}\left(\mathrm{NH}_{2}\right)$. The compounds 4-6 showed an absorption band at $3410-3445 \mathrm{~cm}^{-1}(\mathrm{OH})$, while 10-12 a weak absorption band at $2550-2600 \mathrm{~cm}^{-}$ ${ }^{1}$ (SH). Apart from these the compounds 4-15 exhibited an absorption band at $1665-1680 \mathrm{~cm}^{-1}$ ( $\mathrm{C}=\mathrm{O}$ of pyrimidine / pyrazole ring) while 16-18 at $1740-1750 \mathrm{~cm}^{-1}(\mathrm{C}=\mathrm{O}$ of isoxazole ring). However, all the compounds displayed an absorption band at $1640-1660 \mathrm{~cm}^{-1}$ (Ar-CO). In the ${ }^{1} \mathrm{H}$ NMR spectra of these compounds the methine protons displayed doublets at 4.29-5.45 ppm

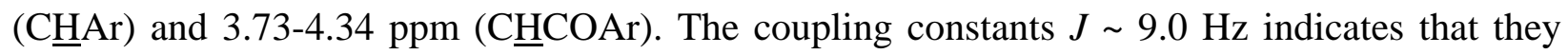
possess trans geometry. Thus the ${ }^{1} \mathrm{H}$ NMR spectra of 4-18 can be rationalized by presuming that the substituents in piperidine, tetrahydropyran and tetrahydrothiopyran rings are in true cis1,3diequatorial arrangement in their preferred rigid chair conformation.14 The substituted pyrimidinone / pyrazolidinone / isoxazolidinone rings which are nearly planar would be perpendicular to the average plane of the heterocyclic rings (Figure).

All the compounds displayed a broad singlet at 6.69-10.12 $\left(\mathrm{NH}_{2} / \mathrm{NH}\right)$, while 4-6 at 6.859.20 ppm (OH). The signals due to $\mathrm{NH}, \mathrm{NH} 2$ and $\mathrm{OH}$ disappeared on deuteration. The compounds 7-9 showed a singlet at 2.70-2.75 $\left(\mathrm{N}-\mathrm{CH}_{3}\right)$, while 10-12 at 1.37-1.42 $\mathrm{ppm}(\mathrm{SH})$.

The structures of all these compounds are also confirmed by ${ }^{13} \mathrm{C}$ NMR spectral data. 


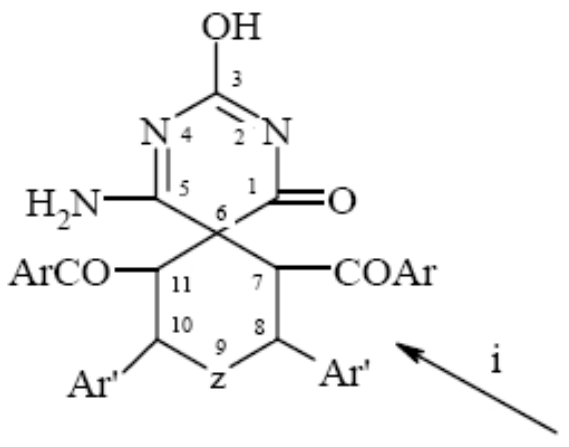

$4 / 5 / 6$<smiles>[Z17]C1[C]([Al])C(C(=O)OCC)C(C#N)(C(=O)OCC)C1OC(=O)CCCC</smiles>

$1 / 2 / 3$<smiles></smiles>

$7 / 8 / 9$

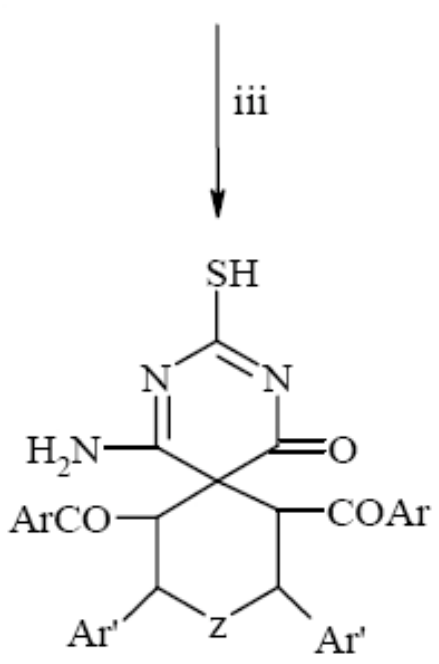<smiles>CCC</smiles>

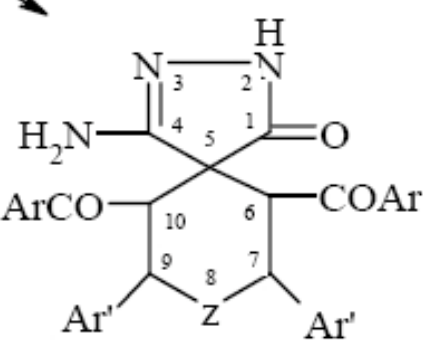

$13 / 14 / 15$

\section{Scheme 1}

$1,4,7,10,13,16 ; Z=N H$

$\mathbf{2 , 5 , 8 , 1 1 , 1 4 , 1 7 ; Z = O}$

$3,6,9,12,15,18 ; Z=S$ ii) $\mathrm{CH}_{3} \mathrm{NHCONHCH} / \mathrm{NaOMe}$

iv) $\mathrm{NH}_{2} \mathrm{NH}_{2} \cdot \mathrm{H}_{2} \mathrm{O} / \mathrm{NaOMe}$ v) $\mathrm{NH}_{2} \mathrm{OH} \cdot \mathrm{HCl} / \mathrm{NaOMe}$

$\begin{array}{lll} & \text { Ar } & \text { Ar' } \\ \text { a } & \mathrm{Ph} & \mathrm{Ph} \\ \text { b } & \mathrm{Ph} & \text { 4-OMe.Ph } \\ \text { c } & \text { 4-Cl.Ph } & \mathrm{Ph}\end{array}$

i) $\mathrm{NH}_{2} \mathrm{CONH}_{2} / \mathrm{NaOMe}$ iii) $\mathrm{NH}_{2} \mathrm{CSNH}_{2} / \mathrm{Na} \mathrm{OMe}$

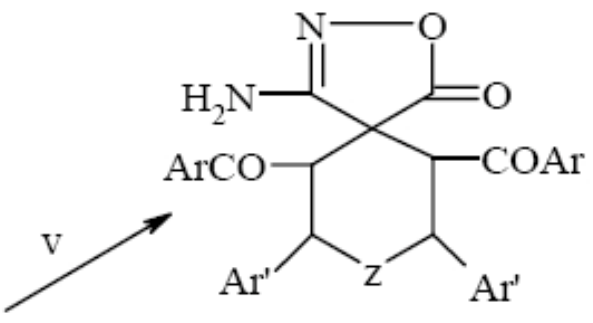

$16 / 17 / 18$

\section{$10 / 11 / 12$}




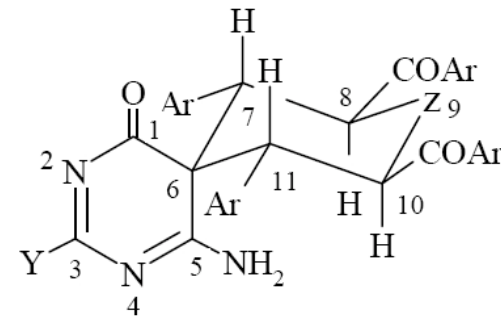

$\mathrm{Z}=\mathrm{NH}, \mathrm{O}, \mathrm{S}$

$\mathrm{Y}=\mathrm{OH}, \mathrm{SH}$

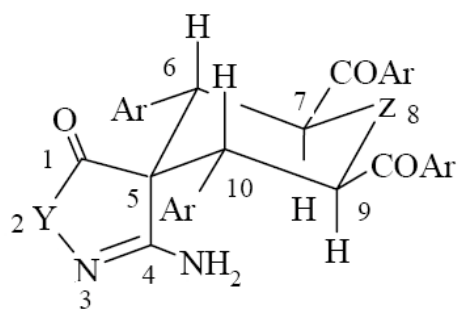

$\mathrm{Z}=\mathrm{NH}, \mathrm{O}, \mathrm{S}$

$\mathrm{Y}=\mathrm{NH}, \mathrm{O}$

\section{Figure 1}

\section{Conclusions}

The versatility of olefinic and gem-cyano, ester functionalities in 1-3 thus forms the basis for the syntheses of novel spiro heterocycles by simple and well-versed methodology.

\section{Antimicrobial activity}

The compounds 4-18 were tested for their antimicrobial activity ${ }^{15}$ at three different concentrations (25, 75 \& $100 \mu \mathrm{g} /$ disc). The antifungal activity was screened on Fusarium solani, Curvularia lunata, and Aspergillus niger using Nystatin (25 $\mu \mathrm{g} / \mathrm{disc})$ as a standard drug and the fungal cultures were grown on potato dextrose broth (PDA) at $25^{\circ} \mathrm{C}$ for $3 \mathrm{~d}$ and finally spore suspension was adjusted to $10^{6}$ spores $/ \mathrm{ml}$. All the compounds were also evaluated for antibacterial activity against Staphylococcus aureus Bacillus subtilis (Gram positive) and Escherichia coli (Gram negative) on nutrient agar plates at $37^{0} \mathrm{C}$ for $24 \mathrm{~h}$ using Gentamycin (25 $\mu \mathrm{g} / \mathrm{disc}$ ) as a reference drug. In general they showed more activity against Gram positive than Gram negative bacteria. Besides, they have also moderately inhibited the growth of fungi. Compounds 4, 6, 13 and 15 showed more inhibitory activity against both bacteria and fungi.

\section{Experimental Section}

General Procedures. Melting points were determined in open capillaries on a Mel-Temp apparatus and are uncorrected. The purity of the compounds was checked by TLC (silica gel H, $\mathrm{BDH}$, ethyl acetate/hexane, 3:1). The IR spectra were recorded on a Perkin-Elmer grating infrared spectrometer, model 337 in $\mathrm{KBr}$ pellets. ${ }^{1} \mathrm{H}$ NMR spectra were recorded in $\mathrm{CDCl}_{3}$ using $300 \mathrm{MHz}$ on a Varian EM-360 spectrometer. ${ }^{13} \mathrm{C}$ NMR spectra were recorded in $\mathrm{CDCl}_{3}$ on a Varian VXR spectrometer operating at $75.5 \mathrm{MHz}$. All chemical shifts were reported in ppm from TMS as an internal standard. The elemental analyses were performed at Punjab University, Chandigarh, India. The compounds 3,5-diaroyl-2,6-diaryl-4-cyano-4-ethoxycarbonyl-piperidines 1, 3,5-diaroyl-2,6-diaryl-4-cyano-4-ethoxy-carbonyl-tetrahydropyrans 2, 3,5-diaroyl-2,6-diaryl4- 
cyano-4-ethoxycarbonyl-tetra-hydrothiopyrans 3 were prepared according to the literature procedure. $^{9}$

5-Amino-7,11-diaroyl-8,10-diaryl-3-hydroxy-2,4,9-triazaspiro[5.5]undeca-2,4-dien-1-ones (4) / 5amino-7,11-diaroyl-8,10-diaryl-3-hydroxy-9-oxa-2,4-diazaspiro[5.5] undeca-2,4-dien1-ones (5) / 5amino-7,11-diaroyl-8,10-diaryl-3-hydroxy-9-thia-2,4-diazaspiro[5.5]undeca2,4-dien-1-ones (6). A mixture of 1 / 2 / 3 (0.526 / 0.607 / 0.628 g, 1 mmol), urea (0.090 g, 1.5 mmol), MeOH (20 mL) and 10\% $\mathrm{NaOMe}(5 \mathrm{~mL})$ was refluxed for 6-7 h. The solution was cooled and poured onto crushed ice containing $\mathrm{HCl}$. The solid obtained was filtered, dried and recrystallized from $\mathrm{MeOH}$.

4a. (0.373 g, 67\%); mp 261-263 ${ }^{\circ} \mathrm{C}$; Anal. Calcd. $\mathrm{C}_{34} \mathrm{H}_{28} \mathrm{~N}_{4} \mathrm{O}_{4}$ : C, 73.37; H, 5.07; N, 10.07 . Found: C, 73.49; H, 5.02; N, 10.17. IR (KBr, $\left.\mathrm{cm}^{-1}\right) 3212$ \& $3264\left(\mathrm{NH}_{2}\right), 3300(\mathrm{NH}), 3420(\mathrm{OH})$, 1640 (OAr), 1670 (CO at 1/1,3); ${ }^{1} \mathrm{H}-\mathrm{NMR}\left(300 \mathrm{MHz}, \mathrm{CDCl}_{3}\right) \delta 3.81\left(2 \mathrm{H}, \mathrm{C}_{7} \& \mathrm{C}_{11}-\mathrm{H}, \mathrm{d}, J=\right.$ $9.0 \mathrm{~Hz}), 4.59\left(2 \mathrm{H}, \mathrm{C}_{8} \& \mathrm{C}_{10}-\mathrm{H}, \mathrm{d}, J=9.0 \mathrm{~Hz}\right), 7.05-7.87(20 \mathrm{H}, \mathrm{Ar}-\mathrm{H}, \mathrm{m}), 6.69$ (2H, bs, $\left.\mathrm{NH}_{2}\right)$, 6.88 (1H, bs, OH) 10.12 (1H, bs, NH); ${ }^{13} \mathrm{C}-\mathrm{NMR}$ (75.5 MHz, $\mathrm{CDCl}_{3}$ ) $\delta 36.3$ (C-6), 44.7 (C-7 \& C-11), 48.9 (C-8 \& C-10), 159.6 (C-3), 164.0 (C-5), 200.7 (C=O), 204.0 (C-1).

4b. (0.382 g, 62\%); mp 274-276 ${ }^{\circ} \mathrm{C}$; Anal. Calcd. $\mathrm{C}_{36} \mathrm{H}_{32} \mathrm{~N}_{4} \mathrm{O}_{6}$ : C, 70.12; H, 5.23; N, 9.09. Found: C, 70.22; H, 5.26; N, 9.18. IR (KBr, cm $\left.{ }^{-1}\right) 3220 \& 3275\left(\mathrm{NH}_{2}\right), 3298(\mathrm{NH}), 3430(\mathrm{OH}), 1645$ ( $\underline{\mathrm{COAr}}$ ), 1665 (CO at 1/1,3); ${ }^{1} \mathrm{H}-\mathrm{NMR}\left(300 \mathrm{MHz}, \mathrm{CDCl}_{3}\right) \delta 3.69$ (6H, s, Ar-OCH $), 3.85$ (d, C \& $\left.\mathrm{C}_{11}-\mathrm{H}, 2 \mathrm{H}, J=9.2 \mathrm{~Hz}\right), 4.52\left(2 \mathrm{H}, \mathrm{C}_{8} \& \mathrm{C}_{10}-\mathrm{H}, \mathrm{d}, J=9.2 \mathrm{~Hz}\right), 7.14-7.83$ (18H, m, Ar-H), 6.71 (2H, $\mathrm{NH}_{2}$, bs), 6.85 (1H, OH, bs), 10.08 (1H, NH, bs); ${ }^{13} \mathrm{C}-\mathrm{NMR} 36.8$ (C-6), 45.2 (C-7 \& C-11), 49.0 (C-8 \& C-10), $56.0\left(\mathrm{OCH}_{3}\right), 160.0$ (C-3), 163.1 (C-5), 201.3 (C=O), 204.9 (C-1).

4c. (0.350 g, 59\%); mp 278-280 ${ }^{\circ}$; Anal. Calcd. $\mathrm{C}_{34} \mathrm{H}_{26} \mathrm{C}_{12} \mathrm{~N}_{4} \mathrm{O}_{4}$ : C, 65.29; H, 4.19; N, 8.96. Found: C, 65.36; H, 4.15; N, 8.90. IR ( KBr, cm $\left.{ }^{-1}\right) 3222$ \& $3275\left(\mathrm{NH}_{2}\right), 3304(\mathrm{NH}), 3440(\mathrm{OH})$, 1650 (OAr), 1675 (CO at 1/1,3); ${ }^{1} \mathrm{H}-\mathrm{NMR}\left(300 \mathrm{MHz}, \mathrm{CDCl}_{3}\right) \delta 3.86\left(2 \mathrm{H}, \mathrm{C}_{7} \& \mathrm{C}_{11}-\mathrm{H}, \mathrm{d}, J=\right.$ $9.0 \mathrm{~Hz}), 4.54\left(2 \mathrm{H}, \mathrm{C}_{8} \& \mathrm{C}_{10}-\mathrm{H}, \mathrm{d}, J=9.0 \mathrm{~Hz}\right), 7.12-7.81(18 \mathrm{H}, \mathrm{Ar}-\mathrm{H}, \mathrm{m}), 6.72$ (2H, NH , bs), $6.87(1 \mathrm{H}, \mathrm{OH}, \mathrm{bs}), 10.06(1 \mathrm{H}, \mathrm{NH}, \mathrm{bs}) ;{ }^{13} \mathrm{C}-\mathrm{NMR}\left(75.5 \mathrm{MHz}, \mathrm{CDCl}_{3}\right) \delta 36.3(\mathrm{C}-6), 45.4(\mathrm{C}-7$ \& C-11), 49.4 (C-8 \& C-10), 159.6 (C-3), 163.2 (C-5), 201.7 (C=O), 204.4 (C-1).

5a. (0.346 g, 68\%); mp 290-292 ${ }^{\circ} \mathrm{C}$; Anal. Calcd. $\mathrm{C}_{34} \mathrm{H}_{27} \mathrm{~N}_{3} \mathrm{O}_{5}$ : C, 73.24; H, 4.88; N, 7.54. Found:C, 73.10; H, 4.82; N, 7.64. IR (KBr, cm $\left.{ }^{-1}\right) 3200$ \& $3268\left(\mathrm{NH}_{2}\right), 3410(\mathrm{OH}), 1645$ (COAr), 1665 (CO at 1/1,3); ${ }^{1} \mathrm{H}-\mathrm{NMR}\left(300 \mathrm{MHz} \mathrm{CDCl}_{3}\right) \delta 3.90\left(2 \mathrm{H}, \mathrm{C}_{7} \& \mathrm{C}_{11}-\mathrm{H}, \mathrm{d}, J=9.1 \mathrm{~Hz}\right), 5.43$ $\left(2 \mathrm{H}, \mathrm{C}_{8} \& \mathrm{C}_{10}-\mathrm{H}, \mathrm{d}, J=9.1 \mathrm{~Hz}\right), 7.20-7.90(20 \mathrm{H}, \mathrm{Ar}-\mathrm{H}, \mathrm{m}), 6.88\left(2 \mathrm{H}, \mathrm{NH}_{2}, \mathrm{bs}\right), 8.34(1 \mathrm{H}, \mathrm{OH}$, bs); ${ }^{13} \mathrm{CNMR}\left(75.5 \mathrm{MHz}, \mathrm{CDCl}_{3}\right) \delta 37.1$ (C-6), 45.5 (C-7 \& C-11), 66.1 (C-8 \& C-10), 159.2 (C3), 163.1 (C-5), 201.7 (C=O), 202.6 (C-1).

5b. (0.401 g, 65\%); mp 265-267 ${ }^{\circ} \mathrm{C}$; Anal. Calcd. $\mathrm{C}_{36} \mathrm{H}_{31} \mathrm{~N}_{3} \mathrm{O}_{7}$ : C, 70.06; H, 5.05; N, 6.80 .

Found: C, 70.17; H, 5.10; N, 6.89. IR (KBr, $\left.\mathrm{cm}^{-1}\right) 3212$ \& $3278\left(\mathrm{NH}_{2}\right), 3420(\mathrm{OH}), 1640$ ( $\underline{\mathrm{COAr}}$ ), 1668 (CO at 1/1,3); ${ }^{1} \mathrm{H}-\mathrm{NMR}\left(300 \mathrm{MHz}, \mathrm{CDCl}_{3}\right) \delta 3.68\left(6 \mathrm{H}, \mathrm{Ar}_{-} \mathrm{OCH}_{3}, \mathrm{~s}\right), 3.85\left(2 \mathrm{H}, \mathrm{C}_{7}\right.$ \& $\left.\mathrm{C}_{11}-\mathrm{H}, \mathrm{d}, J=9.2 \mathrm{~Hz}\right), 5.40\left(2 \mathrm{H}, \mathrm{C}_{8} \& \mathrm{C}_{10}-\mathrm{H}, \mathrm{d}, J=9.2 \mathrm{~Hz}\right), 7.18-7.88(18 \mathrm{H}, \mathrm{Ar}-\mathrm{H}, \mathrm{m}), 6.86$ (2H, $\mathrm{NH}_{2}$, bs), 8.32 (1H, OH, bs); ${ }^{13} \mathrm{C}-\mathrm{NMR}\left(75.5 \mathrm{MHz}, \mathrm{CDCl}_{3}\right.$ ) $\delta 37.4$ (C-6), 45.4 (C-7 \& C11), $56.0\left(\mathrm{OCH}_{3}\right), 65.6$ (C-8 \& C-10), 159.4 (C-3), 162.5 (C-5), 201.5 (C=O), 202.6 (C-1). 
5c. (0.426 g, 66\%); mp 274-276 ${ }^{\circ} \mathrm{C}$; Anal. Calcd. $\mathrm{C}_{34} \mathrm{H}_{25} \mathrm{C}_{12} \mathrm{~N}_{3} \mathrm{O}_{5}$ : C, 65.18; H, 4.02; N, 6.70. Found: C, 65.27; H, 4.05; N, 6.76. IR (KBr, cm $\left.{ }^{-1}\right) 3215$ \& $3280\left(\mathrm{NH}_{2}\right), 3445(\mathrm{OH}), 1660$ (COAr), 1670 (CO at 1/1,3); ${ }^{1} \mathrm{H}-\mathrm{NMR}\left(300 \mathrm{MHz}, \mathrm{CDCl}_{3}\right) \delta 3.87\left(2 \mathrm{H}, \mathrm{C}_{7} \& \mathrm{C}_{11}-\mathrm{H}, \mathrm{d}, J=9.4\right.$ $\mathrm{Hz}), 5.42\left(2 \mathrm{H}, \mathrm{C}_{8} \& \mathrm{C}_{10}-\mathrm{H}, \mathrm{d}, J=9.4 \mathrm{~Hz}\right), 7.1-7.87$ (18H, Ar-H, m), 6.88 (2H, NH 2 , bs), 8.24 (1H, OH, bs); ${ }^{13} \mathrm{C}-\mathrm{NMR}\left(75.5 \mathrm{MHz}, \mathrm{CDCl}_{3}\right) \delta 36.5$ (C-6), 47.0 (C-7 \& C-11), 67.4 (C-8 \& C10), 160.1 (C-3), 164.7 (C-5), 200.3 (C=O), 205.5 (C-1).

6a. (0.356 g, 62\%); mp 272-274 ${ }^{\circ} \mathrm{C}$; Anal. Calcd. $\mathrm{C}_{34} \mathrm{H}_{27} \mathrm{~N}_{3} \mathrm{O}_{4} \mathrm{~S}$ : C, 71.19; H, 4.74; N, 7.32 . Found: C, 71.11; H, 4.77; N, 7.28. IR (KBr, cm $\left.{ }^{-1}\right) 3235$ \& $3300\left(\mathrm{NH}_{2}\right), 3415(\mathrm{OH}), 1640$ ( $\underline{\mathrm{CO}} \mathrm{Ar}$ ), 1672 (CO at 1/1,3); ${ }^{1} \mathrm{H}-\mathrm{NMR}\left(300 \mathrm{MHz}, \mathrm{CDCl}_{3}\right) \delta 4.01\left(2 \mathrm{H}, \mathrm{C}_{7} \& \mathrm{C}_{11}-\mathrm{H}, \mathrm{d}, J=9.1\right.$ $\mathrm{Hz}), 4.29$ (2H, $\left.\mathrm{C}_{8} \& \mathrm{C}_{10}-\mathrm{H}, \mathrm{d}, J=9.1 \mathrm{~Hz}\right), 6.69$ (2H, NH , bs), 7.08-7.90 (20H, Ar-H, m), 9.20 (1H, OH, bs); ${ }^{13} \mathrm{C}-\mathrm{NMR}\left(75.5 \mathrm{MHz}, \mathrm{CDCl}_{3}\right) \delta 32.2$ (C-8 \& C-10), 38.2 (C-6), 45.9 (C-7 \& C11), 161.9 (C-3), 162.7 (C-5), 201.1 (C=O), 203.6 (C-1).

6b. (0.374 g, 59\%); mp 278-280 ${ }^{\circ} \mathrm{C}$; Anal. Calcd. $\mathrm{C}_{36} \mathrm{H}_{31} \mathrm{~N}_{3} \mathrm{O}_{6} \mathrm{~S}$ : C, 68.23; H, 4.93; N, 6.63. Found: C, 68.12; H, 4.90; N, 6.70. IR (KBr, $\left.\mathrm{cm}^{-1}\right) 3240$ \& $3305\left(\mathrm{NH}_{2}\right), 3420(\mathrm{OH}), 1645$ ( $\underline{\mathrm{CO}} \mathrm{Ar}$ ), 1665 (CO at 1/1,3); ${ }^{1} \mathrm{H}-\mathrm{NMR}\left(300 \mathrm{MHz}, \mathrm{CDCl}_{3}\right) \delta 3.73\left(6 \mathrm{H}, \mathrm{Ar}-\mathrm{OCH}_{3}, \mathrm{~s}\right), 4.04\left(2 \mathrm{H}, \mathrm{C}_{7}\right.$ \& $\left.\mathrm{C}_{11}-\mathrm{H}, \mathrm{d}, J=9.5 \mathrm{~Hz}\right), 4.32\left(2 \mathrm{H}, \mathrm{C}_{8} \& \mathrm{C}_{10}-\mathrm{H}, \mathrm{d}, J=9.5 \mathrm{~Hz}\right), 6.73\left(2 \mathrm{H}, \mathrm{NH}_{2}\right.$, bs), 7.09-7.89 (18H, Ar-H, m), 9.16 (1H, OH, bs); ${ }^{13} \mathrm{C}-\mathrm{NMR}$ (75.5 MHz, $\mathrm{CDCl}_{3}$ ) $\delta 30.8$ (C-8 \& C-10), 37.8 (C6), 45.2 (C-7 \& C-11), $57.9\left(\mathrm{OCH}_{3}\right), 160.5$ (C-3), 164.5 (C-5), 199.3 (C=O), 204.1 (C-1).

6c. (0.418 g, 65\%); mp 262-264 ${ }^{\circ} \mathrm{C}$; Anal. Calcd. $\mathrm{C}_{34} \mathrm{H}_{25} \mathrm{C}_{12} \mathrm{~N}_{3} \mathrm{O}_{4} \mathrm{~S}$ : C, 63.55; H, 3.92; N, 6.54. Found: C, 63.61; H, 3.89; N, 6.50. IR (KBr, cm $\left.{ }^{-1}\right) 3235$ \& $3310\left(\mathrm{NH}_{2}\right), 3440(\mathrm{OH}), 1655$ (COAr), 1680 (CO at 1/1,3); ${ }^{1} \mathrm{H}-\mathrm{NMR}\left(300 \mathrm{MHz}, \mathrm{CDCl}_{3}\right) \delta 4.07\left(2 \mathrm{H}, \mathrm{C}_{7} \& \mathrm{C}_{11}-\mathrm{H}, \mathrm{d}, J=9.3\right.$ $\mathrm{Hz}$ ), 4.34 (2H, $\left.\mathrm{C}_{8} \& \mathrm{C}_{10}-\mathrm{H}, \mathrm{d}, J=9.3 \mathrm{~Hz}\right), 6.70$ (2H, NH , bs), 7.10-7.90 (18H, Ar-H, m), 9.14 (1H, OH, bs); ${ }^{13} \mathrm{C}-\mathrm{NMR}\left(75.5 \mathrm{MHz}, \mathrm{CDCl}_{3}\right) \delta 30.4$ (C-8 \& C-10), 36.7 (C-6), 45.8 (C-7 \& C11), 162.0 (C-3), 164.9 (C-5), 197.9 (C=O), 204.2 (C-1).

7,11-Diaroyl-8,10-diaryl-2,4-dimethyl-5-imino-2,4,9-triazaspiro[5.5]undecane-1,3-diones (7) / 7,11diaroyl-8,10-diaryl-2,4-dimethyl-5-imino-9-oxa-2,4-diazaspiro[5.5] undecane-1,3diones (8) / 7,11diaroyl-8,10-diaryl-2,4-dimethyl-5-imino-9-thia-2,4diazaspiro[5.5] undecane-1,3-diones (9). The compound 1 / 2 / 3 (0.526 / 0.607 / 0.628 g, 1 mmol), N,N'-dimethyl urea (0.089 g, 1 mmol), 10 \% NaOMe $(5 \mathrm{~mL})$ in dry $\mathrm{MeOH}(20 \mathrm{~mL})$ was refluxed for 9-13 h. Then, it was cooled and poured onto crushed ice containing conc. $\mathrm{HCl}$. The separated solid was collected by filtration, dried and recrystallized from $\mathrm{MeOH}$.

7a. (0.362 g, 62\%); mp 266-268 ${ }^{\circ} \mathrm{C}$; Anal. Calcd. $\mathrm{C}_{36} \mathrm{H}_{32} \mathrm{~N}_{4} \mathrm{O}_{4}$ : C, 73.95; H, 5.51; N, 9.58. Found:

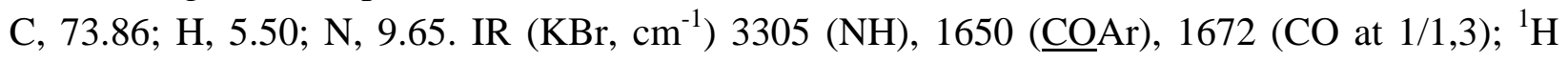
NMR (300 MHz, $\left.\mathrm{CDCl}_{3}\right) \delta 2.71\left(6 \mathrm{H}, \mathrm{N}-\mathrm{CH}_{3}, \mathrm{~s}\right), 3.81\left(2 \mathrm{H}, \mathrm{C}_{7} \& \mathrm{C}_{11}-\mathrm{H}, \mathrm{d}, J=9.2 \mathrm{~Hz}\right), 4.30(2 \mathrm{H}$, $\mathrm{C}_{8} \& \mathrm{C}_{10}-\mathrm{H}, \mathrm{d}, J=9.2 \mathrm{~Hz}$ ), 7.08-7.89 (20H, Ar-H, m), 9.24 (2H, NH, bs); ${ }^{13} \mathrm{C}-\mathrm{NMR}(75.5 \mathrm{MHz}$, $\left.\mathrm{CDCl}_{3}\right) \delta 28.2 \& 29.4\left(\mathrm{~N}^{-\mathrm{CH}_{3}}\right), 34.3$ (C-6), 42.7 (C-7 \& C-11), 44.6 (C-8 \& C-10), 158.0 (C-3), 164.0 (C-5), 176.8 (C-1), $200.7(\mathrm{C}=\mathrm{O})$.

7b. (0.387 g, 60\%); mp 258-260 ${ }^{\circ} \mathrm{C}$; Anal. Calcd. $\mathrm{C}_{38} \mathrm{H}_{36} \mathrm{~N}_{4} \mathrm{O}_{6}$ : C, 70.79; H, 5.63; N, 8.69. Found: C, 70.72; H, 5.58; N, 8.79. IR (KBr, $\left.\mathrm{cm}^{-1}\right) 3312$ (NH), 1645 ( $\left.\underline{\mathrm{CO} A r}\right), 1672$ (CO at 1/1,3); ${ }^{1} \mathrm{H}-$ 
NMR (300 MHz, $\left.\mathrm{CDCl}_{3}\right) \delta 2.70\left(6 \mathrm{H}, \mathrm{NCH}_{3}, \mathrm{~s}\right), 3.74\left(6 \mathrm{H}, \mathrm{Ar}-\mathrm{OCH}_{3}, \mathrm{~s}\right), 3.83\left(2 \mathrm{H}, \mathrm{C}_{7} \& \mathrm{C}_{11}-\mathrm{H}, \mathrm{d}\right.$, $J=9.4 \mathrm{~Hz}$ ), 4.32 (2H, C8 \& $\mathrm{C}_{10}-\mathrm{H}, \mathrm{d}, J=9.4 \mathrm{~Hz}$ ), 7.15-7.89 (18H, Ar-H, m), 9.26 (2H, NH, bs);

${ }^{13} \mathrm{C}-\mathrm{NMR}\left(75.5 \mathrm{MHz}, \mathrm{CDCl}_{3}\right) \delta 29.7 \& 28.2\left(\mathrm{~N}^{\left.-\mathrm{CH}_{3}\right),} 33.4(\mathrm{C}-6), 41.9\right.$ (C-7 \& C-11), 45.0 (C-8 \& C-10), $56.0\left(\mathrm{OCH}_{3}\right), 160.1$ (C-3), 163.1 (C-5), $174.1(\mathrm{C}-1), 202.1(\mathrm{C}=\mathrm{O})$.

7c. (0.431 g, 66\%); mp 264-266 ${ }^{\circ} \mathrm{C}$; Anal. Calcd. $\mathrm{C}_{36} \mathrm{H}_{30} \mathrm{C}_{12} \mathrm{~N}_{4} \mathrm{O}_{4}$ : C, 66.16; H, 4.63; N, 8.57. Found: C, 66.08; H, 4.67; N, 8.63. IR (KBr, cm $\left.{ }^{-1}\right) 3308$ (NH), 1640 (OAr), 1665 (CO at 1/1,3); ${ }^{1} \mathrm{H}-\mathrm{NMR}\left(300 \mathrm{MHz}, \mathrm{CDCl}_{3}\right) \delta 2.72\left(6 \mathrm{H}, \mathrm{N}-\mathrm{CH}_{3}, \mathrm{~s}\right), 3.82\left(2 \mathrm{H}, \mathrm{C}_{7} \& \mathrm{C}_{11}-\mathrm{H}, \mathrm{d}, J=9.3 \mathrm{~Hz}\right), 4.35$ (2H, $\left.\mathrm{C}_{8} \& \mathrm{C}_{10}-\mathrm{H}, \mathrm{d}, J=9.3 \mathrm{~Hz}\right), 7.16-7.91$ (18H, Ar-H, m), 9.28 (2H, NH, bs); ${ }^{13} \mathrm{C}-\mathrm{NMR}(75.5$ $\left.\mathrm{MHz}, \mathrm{CDCl}_{3}\right) \delta 28.9 \& 28.8\left(\mathrm{~N}-\mathrm{CH}_{3}\right), 33.1(\mathrm{C}-6), 42.5$ (C-7 \& C-11), 45.4 (C-8 \& C-10), 161.5 (C-3), 164.4 (C-5), 176.4 (C-1), 204.1 (C=O).

8a. (0.340 g, 58\%); mp 189-191 ${ }^{\circ} \mathrm{C}$; Anal. Calcd. $\mathrm{C}_{36} \mathrm{H}_{31} \mathrm{~N}_{3} \mathrm{O}_{5}$ : C, 73.83; H, 5.34; N, 7.17. Found: C, 73.93; H, 5.37; N, 7.12. IR (KBr, cm ${ }^{-1}$ ) 3298 (NH), 1660 ( $\underline{\mathrm{CO} A r), ~} 1668$ (CO at 1/1,3); ${ }^{1} \mathrm{H}$ NMR (300 MHz, $\left.\mathrm{CDCl}_{3}\right) \delta 2.74\left(6 \mathrm{H}, \mathrm{N}-\mathrm{CH}_{3}, \mathrm{~s}\right), 3.93\left(2 \mathrm{H}, \mathrm{C}_{7} \& \mathrm{C}_{11}-\mathrm{H}, \mathrm{d}, J=9.1 \mathrm{~Hz}\right), 5.44(2 \mathrm{H}$, $\left.\mathrm{C}_{8} \& \mathrm{C}_{10}-\mathrm{H}, \mathrm{d}, J=9.1 \mathrm{~Hz}\right), 7.20-7.90$ (20H, Ar-H, m), 9.19 (1H, NH, bs); ${ }^{13} \mathrm{C}-\mathrm{NMR}(75.5 \mathrm{MHz}$, $\left.\mathrm{CDCl}_{3}\right) \delta 29.9 \& 28.5\left(\mathrm{~N}-\mathrm{CH}_{3}\right), 33.5$ (C-6), 41.3 (C-7 \& C-11), 65.9 (C-8 \& C-10), 157.4 (C-3), 162.3 (C-5), 175.0 (C-1), $203.1(\mathrm{C}=\mathrm{O})$.

8b. (0.420 g, 64\%); mp 200-202 ${ }^{\circ} \mathrm{C}$; Anal. Calcd. $\mathrm{C}_{38} \mathrm{H}_{35} \mathrm{~N}_{3} \mathrm{O}_{7}$ : C, 70.68; H, 5.46; N, 6.51. Found:

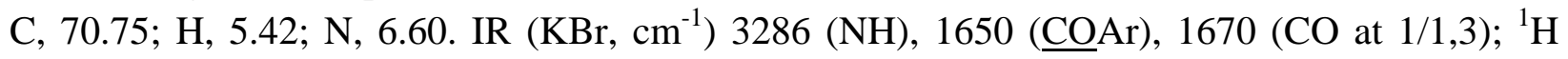
NMR (300 MHz, $\left.\mathrm{CDCl}_{3}\right) \delta 2.71\left(6 \mathrm{H}, \mathrm{N}-\mathrm{CH}_{3}, \mathrm{~s}\right), 3.74\left(6 \mathrm{H}, \mathrm{Ar}-\mathrm{OCH}_{3}, \mathrm{~s}\right), 3.92\left(2 \mathrm{H}, \mathrm{C}_{7} \& \mathrm{C}_{11}-\mathrm{H}\right.$, d, $J=9.4 \mathrm{~Hz}$ ), $5.42\left(2 \mathrm{H}, \mathrm{C}_{8} \& \mathrm{C}_{10}-\mathrm{H}, \mathrm{d}, J=9.4 \mathrm{~Hz}\right), 7.18-7.90(18 \mathrm{H}, \mathrm{Ar}-\mathrm{H}, \mathrm{m}), 9.20(1 \mathrm{H}, \mathrm{NH}$, bs); ${ }^{13} \mathrm{C}-\mathrm{NMR}\left(75.5 \mathrm{MHz}, \mathrm{CDCl}_{3}\right) \delta 29.3 \& 26.8\left(\mathrm{~N}-\mathrm{CH}_{3}\right), 34.3(\mathrm{C}-6), 42.1(\mathrm{C}-7$ \& $\mathrm{C}-11), 56.0$ $\left(\mathrm{OCH}_{3}\right), 65.6$ (C-8 \& C-10), 156.7 (C-3), 163.3 (C-5), 175.4 (C-1), 205.3 (C=O).

8c. (0.410 g, 65\%); mp 215-217 ${ }^{\circ} \mathrm{C}$; Anal. Calcd. $\mathrm{C}_{36} \mathrm{H}_{29} \mathrm{C}_{12} \mathrm{~N}_{3} \mathrm{O}_{5}$ : C, 66.06; H, 4.47; N, 6.42. Found: C, 66.16; H, 4.45; N, 6.34. IR (KBr, cm $\left.{ }^{-1}\right) 3304$ (NH), 1640 (COAr), 1675 (CO at 1/1,3); ${ }^{1} \mathrm{H}-\mathrm{NMR}\left(300 \mathrm{MHz}, \mathrm{CDCl}_{3}\right) \delta 2.72\left(6 \mathrm{H}, \mathrm{N}-\mathrm{CH}_{3}, \mathrm{~s}\right), 3.97\left(2 \mathrm{H}, \mathrm{C}_{7} \& \mathrm{C}_{11}-\mathrm{H}, \mathrm{d}, J=9.2 \mathrm{~Hz}\right), 5.44$ (2H, $\left.\mathrm{C}_{8} \& \mathrm{C}_{10}-\mathrm{H}, \mathrm{d}, J=9.2 \mathrm{~Hz}\right), 7.12-7.86$ (18H, Ar-H, m), 9.23 (1H, NH, bs); ${ }^{13} \mathrm{C}-\mathrm{NMR}(75.5$ $\left.\mathrm{MHz}, \mathrm{CDCl}_{3}\right) \delta 29.3$ \& $28.0\left(\mathrm{~N}^{\left.-\mathrm{CH}_{3}\right),} 32.1\right.$ (C-6), 40.5 (C-7 \& C-11), 66.7 (C-8 \& C-10), 156.0 (C-3), 161.9 (C-5), 177.5 (C-1), $202.4(\mathrm{C}=\mathrm{O})$.

9a. (0.391 g, 65\%); mp 255-257 ${ }^{\circ} \mathrm{C}$; Anal. Calcd. $\mathrm{C}_{36} \mathrm{H}_{31} \mathrm{~N}_{3} \mathrm{O}_{4} \mathrm{~S}$ : C, 71.86; H, 5.19; N, 6.98 . Found: C, 71.98; H, 5.13; N, 7.06. IR (KBr, cm $\left.{ }^{-1}\right) 3306$ (NH), 1645 (COAr), 1670 (CO at 1/1,3); ${ }^{1} \mathrm{H}-\mathrm{NMR}\left(300 \mathrm{MHz}, \mathrm{CDCl}_{3}\right) \delta 2.75\left(6 \mathrm{H}, \mathrm{N}-\mathrm{CH}_{3}, \mathrm{~s}\right), 3.98\left(2 \mathrm{H}, \mathrm{C}_{7} \& \mathrm{C}_{11}-\mathrm{H}, \mathrm{d}, J=9.1 \mathrm{~Hz}\right), 4.32$ (2H, $\left.\mathrm{C}_{8} \& \mathrm{C}_{10}-\mathrm{H}, \mathrm{d}, J=9.1 \mathrm{~Hz}\right), 7.12-7.89$ (20H, Ar-H, m), 9.09 (1H, NH, bs); ${ }^{13} \mathrm{C}-\mathrm{NMR}(75.5$ $\left.\mathrm{MHz}, \mathrm{CDCl}_{3}\right) \delta 28.1 \& 29.7\left(\mathrm{~N}-\mathrm{CH}_{3}\right), 33.8$ (C-8 \& C-10), 36.5 (C-6), 43.5 (C-7 \& C-11), 158.0 (C-3), 164.9 (C-5), 176.0 (C-1), $201.1(\mathrm{C}=\mathrm{O})$.

9b. (0.463 g, 70\%); mp 283-285 ${ }^{\circ} \mathrm{C}$; Anal. Calcd. $\mathrm{C}_{38} \mathrm{H}_{35} \mathrm{~N}_{3} \mathrm{O}_{6} \mathrm{~S}$ : C, 68.97; H, 5.33; N, 6.35 . Found: C, 69.06; H, 5.35; N, 6.30. IR (KBr, cm $\left.{ }^{-1}\right) 3296$ (NH), 1650 ( $\underline{\mathrm{COAr}}$ ), 1675 (CO at 1/1,3); ${ }^{1} \mathrm{H}-\mathrm{NMR}\left(300 \mathrm{MHz}, \mathrm{CDCl}_{3}\right) \delta 2.72\left(6 \mathrm{H}, \mathrm{N}-\mathrm{CH}_{3}, \mathrm{~s}\right), 3.77\left(6 \mathrm{H}, \mathrm{Ar}-\mathrm{OCH}_{3}, \mathrm{~s}\right), 4.06\left(2 \mathrm{H}, \mathrm{C}_{7} \& \mathrm{C}_{11^{-}}\right.$ $\mathrm{H}, \mathrm{d}, J=9.0 \mathrm{~Hz}$ ), $4.32\left(2 \mathrm{H}, \mathrm{C}_{8} \& \mathrm{C}_{10}-\mathrm{H}, \mathrm{d}, J=9.0 \mathrm{~Hz}\right), 7.02-7.83$ (18H, Ar-H, m), $9.24(1 \mathrm{H}$, $\mathrm{NH}$, bs); ${ }^{13} \mathrm{C}-\mathrm{NMR}\left(75.5 \mathrm{MHz}, \mathrm{CDCl}_{3}\right) \delta 28.9 \& 29.2\left(\mathrm{~N}-\mathrm{CH}_{3}\right), 32.2$ (C-8 \& C-10), $35.8(\mathrm{C}-6)$, 42.9 (C-7 \& C-11), $55.9\left(\mathrm{OCH}_{3}\right), 156.6$ (C-3), 163.1 (C-5), 176.5 (C-1), 202.1 (C=O). 
9c. (0.590 g, 68\%); mp 295-297 ${ }^{\circ} \mathrm{C}$; Anal. Calcd. $\mathrm{C}_{36} \mathrm{H}_{29} \mathrm{C}_{12} \mathrm{~N}_{3} \mathrm{O}_{4} \mathrm{~S}$ : C, 64.48; H, 4.36; N, 6.27. Found: C, 64.42; H, 4.40; N, 6.37. IR (KBr, cm $\left.{ }^{-1}\right) 3298$ (NH), 1660 (OA), 1680 (CO at 1/1,3); ${ }^{1} \mathrm{H}-\mathrm{NMR}\left(300 \mathrm{MHz}, \mathrm{CDCl}_{3}\right) \delta 2.74\left(6 \mathrm{H}, \mathrm{N}-\mathrm{CH}_{3}, \mathrm{~s}\right), 4.08\left(2 \mathrm{H}, \mathrm{C}_{7} \& \mathrm{C}_{11}-\mathrm{H}, \mathrm{d}, J=9.2 \mathrm{~Hz}\right), 4.33$ (2H, $\left.\mathrm{C}_{8} \& \mathrm{C}_{10}-\mathrm{H}, \mathrm{d}, J=9.2 \mathrm{~Hz}\right), 7.10-7.85$ (18H, Ar-H, m), 9.28 (1H, NH, bs); ${ }^{13} \mathrm{C}-\mathrm{NMR}(75.5$ $\left.\mathrm{MHz}, \mathrm{CDCl}_{3}\right) \delta 27.8$ \& $29.2\left(\mathrm{~N}-\mathrm{CH}_{3}\right), 34.7$ (C-8 \& C-10), $35.6(\mathrm{C}-6), 43.5$ (C-7 \& C-11), 154.7 (C-3), 163.6 (C-5), 174.8 (C-1), $202.5(\mathrm{C}=\mathrm{O})$.

5-Amino-7,11-diaroyl-8,10-diaryl-3-mercapto-2,4,9-triazaspiro[5.5]undeca-2,4-dien-1-ones (10) / 5amino-7,11-diaroyl-8,10-diaryl-3-mercapto-9-oxa-2,4-diazaspiro[5.5] undeca-2,4dien-1-ones (11) / 5amino-7,11-diaroyl-8,10-diaryl-3-mercapto-9-thia-2,4diazaspiro[5.5]undeca-2,4-dien-1-ones (12). A mixture of 1 / 2 / 3 (0.526 / 0.607 / 0.628 g, 1 mmol), thiourea (0.074 g, $1 \mathrm{mmol}$ ), $\mathrm{MeOH}$ (20 mL) and 10\% NaOMe $(5 \mathrm{~mL})$ was refluxed for $11-13 \mathrm{~h}$. The solution was cooled and poured onto crushed ice containing $\mathrm{HCl}$. The solid obtained was filtered, dried and recrystallized from $\mathrm{MeOH}$.

10a. (0.349 g, 61\%); mp 208-210 ${ }^{\circ}$; Anal. Calcd. $\mathrm{C}_{34} \mathrm{H}_{28} \mathrm{~N}_{4} \mathrm{O}_{3} \mathrm{~S}$ : C, 71.31; H, 4.93; N, 9.78. Found: C, 71.25; H, 4.99; N, 9.70. IR (KBr, $\left.\mathrm{cm}^{-1}\right) 3210$ \& $3275\left(\mathrm{NH}_{2}\right), 3312(\mathrm{NH}), 1640$ (COAr), 1665 (CO at 1/1,3); ${ }^{1} \mathrm{H}-\mathrm{NMR}\left(300 \mathrm{MHz}, \mathrm{CDCl}_{3}\right) \delta 1.38$ (1H, SH, s), $3.84\left(2 \mathrm{H}, \mathrm{C}_{7} \&\right.$ $\left.\mathrm{C}_{11}-\mathrm{H}, \mathrm{d}, J=9.4 \mathrm{~Hz}\right), 4.57\left(2 \mathrm{H}, \mathrm{C}_{8} \& \mathrm{C}_{10}-\mathrm{H}, \mathrm{d}, J=9.4 \mathrm{~Hz}\right), 7.06-7.87$ (20H, Ar-H, m), 9.23 (3H, $\mathrm{NH}, \mathrm{NH} 2$, bs); ${ }^{13} \mathrm{C}-\mathrm{NMR}\left(75.5 \mathrm{MHz}, \mathrm{CDCl}_{3}\right) \delta 35.1$ (C-6), 42.6 (C-7 \& C-11), 43.1 (C-8 \& C10), 162.8 (C-3), 163.1 (C-5), 200.7 (C=O), 204.1 (C-1).

10b. (0.405 g, 64\%); mp 198-200 ${ }^{\circ} \mathrm{C}$; Anal. Calcd. $\mathrm{C}_{36} \mathrm{H}_{32} \mathrm{~N}_{4} \mathrm{O}_{5} \mathrm{~S}$ : C, 68.34; H, 5.10; N, 8.85 . Found: C, 68.43; H, 5.05; N, 8.92. IR (KBr, $\left.\mathrm{cm}^{-1}\right) 3220$ \& $3268\left(\mathrm{NH}_{2}\right), 3300(\mathrm{NH}), 1645$ ( $\underline{\text { COAr }), ~} 1672$ (CO at 1/1,3); ${ }^{1} \mathrm{H}-\mathrm{NMR}\left(300 \mathrm{MHz}, \mathrm{CDCl}_{3}\right) \delta 1.36(1 \mathrm{H}, \mathrm{SH}, \mathrm{s}), 3.67$ (6H, Ar$\left.\mathrm{OCH}_{3}, \mathrm{~s}\right), 3.82\left(2 \mathrm{H}, \mathrm{C}_{7} \& \mathrm{C}_{11}-\mathrm{H}, \mathrm{d}, J=9.2 \mathrm{~Hz}\right), 4.55$ (d, 2H, $\left.\mathrm{C}_{8} \& \mathrm{C}_{10}-\mathrm{H}, J=9.2 \mathrm{~Hz}\right), 7.05-7.88$ (m, 18H, Ar-H), 9.25 (bs, 3H, NH, NH ); ${ }^{13} \mathrm{C}-\mathrm{NMR}$ (75.5 MHz, $\left.\mathrm{CDCl}_{3}\right) \delta 35.4(\mathrm{C}-6), 41.2(\mathrm{C}-7$ \& C-11), 43.6 (C-8 \& C-10), $54.5\left(\mathrm{OCH}_{3}\right), 163.8$ (C-3), 164.3 (C-5), 200.5 (C=O), 205.4 (C-1).

10c. (0.366 g, 57\%); mp 226-228 ${ }^{\circ} \mathrm{C}$; Anal. Calcd. $\mathrm{C}_{34} \mathrm{H}_{26} \mathrm{C}_{22} \mathrm{~N}_{4} \mathrm{O}_{3} \mathrm{~S}$ : C, 63.65; H, 4.08; N, 8.73. Found: C, 63.58; H, 5.00; N, 8.69. IR (KBr, $\left.\mathrm{cm}^{-1}\right) 3222$ \& $3270\left(\mathrm{NH}_{2}\right), 3315(\mathrm{NH}), 1650$ (COAr), 1675 (CO at 1/1,3); ${ }^{1} \mathrm{H}-\mathrm{NMR}\left(300 \mathrm{MHz}, \mathrm{CDCl}_{3}\right) \delta 1.40$ (1H, SH, s), $3.86\left(2 \mathrm{H}, \mathrm{C}_{7} \&\right.$ $\mathrm{C}_{11}-\mathrm{H}, \mathrm{d}, J=9.1 \mathrm{~Hz}$ ), 4.52 (2H, C $8 \mathrm{C}_{10}-\mathrm{H}, \mathrm{d}, J=9.1 \mathrm{~Hz}$ ), 7.02-7.82 (18H, Ar-H, m), 9.27 (3H, $\mathrm{NH}, \mathrm{NH}_{2}$, bs); ${ }^{13} \mathrm{C}-\mathrm{NMR}\left(75.5 \mathrm{MHz}, \mathrm{CDCl}_{3}\right) \delta 34.5$ (C-6), 43.2 (C-7 \& C-11), 43.9 (C-8 \& C10), 164.7 (C-3), 165.3 (C-5), 202.0 (C=O), 203.5 (C-1).

11a. (0.356 g, 62\%); mp 202-204 ${ }^{\circ} \mathrm{C}$; Anal. Calcd. $\mathrm{C}_{34} \mathrm{H}_{27} \mathrm{~N}_{3} \mathrm{O}_{4} \mathrm{~S}$ : C, 71.19; H, 4.74; N, 7.32. Found: C, 71.34; H, 4.70; N, 7.40. IR (KBr, cm $\left.{ }^{-1}\right) 3255$ \& $3320\left(\mathrm{NH}_{2}\right), 1655$ (COAr), 1670 (CO at 1/1,3); ${ }^{1} \mathrm{H}-\mathrm{NMR}\left(300 \mathrm{MHz}, \mathrm{CDCl}_{3}\right) \delta 1.39(1 \mathrm{H}, \mathrm{SH}, \mathrm{s}), 3.75\left(2 \mathrm{H}, \mathrm{C}_{7} \& \mathrm{C}_{11}-\mathrm{H}, \mathrm{d}, J=9.2 \mathrm{~Hz}\right)$, $5.42\left(2 \mathrm{H}, \mathrm{C}_{8} \& \mathrm{C}_{10}-\mathrm{H}, \mathrm{d}, J=9.2 \mathrm{~Hz}\right), 7.19-7.91$ (20H, Ar-H, m), 9.44 (2H, $\mathrm{NH}_{2}$, bs); ${ }^{13} \mathrm{C}-\mathrm{NMR}$ (75.5 MHz, $\mathrm{CDCl}_{3}$ ) $\delta 34.0$ (C-6), 41.4 (C-7 \& C-11), 65.8 (C-8 \& C-10), 163.1 (C-3), 164.9 (C5), $201.0(\mathrm{C}=\mathrm{O}), 203.7$ (C-1).

11b. (0.400 g, 63\%); mp 199-201 ${ }^{\circ} \mathrm{C}$; Anal. Calcd. $\mathrm{C}_{36} \mathrm{H}_{31} \mathrm{~N}_{3} \mathrm{O}_{6} \mathrm{~S}$ : C, 68.23; H, 4.93; N, 6.63. Found: C, 68.33; H, 4.90; N, 6.70. IR (KBr, cm $\left.{ }^{-1}\right) 3265$ \& $3325\left(\mathrm{NH}_{2}\right), 1640$ (OAr), 1674 (CO 
at 1/1,3); ${ }^{1} \mathrm{H}-\mathrm{NMR}\left(300 \mathrm{MHz}, \mathrm{CDCl}_{3}\right) \delta 1.37(1 \mathrm{H}, \mathrm{SH}, \mathrm{s}), 3.69\left(6 \mathrm{H}, \mathrm{Ar}-\mathrm{OCH}_{3}, \mathrm{~s},\right), 3.73\left(2 \mathrm{H}, \mathrm{C}_{7}\right.$ \& $\left.\mathrm{C}_{11}-\mathrm{H}, \mathrm{d}, J=9.1 \mathrm{~Hz}\right), 5.45\left(2 \mathrm{H}, \mathrm{C}_{8} \& \mathrm{C}_{10}-\mathrm{H}, \mathrm{d}, J=9.1 \mathrm{~Hz}\right), 7.15-7.69(18 \mathrm{H}, \mathrm{Ar}-\mathrm{H}, \mathrm{m}), 9.41$ $\left(2 \mathrm{H}, \mathrm{NH}_{2}\right.$, bs); ${ }^{13} \mathrm{C}-\mathrm{NMR}\left(75.5 \mathrm{MHz}, \mathrm{CDCl}_{3}\right) \delta 33.2(\mathrm{C}-6), 41.8$ (C-7 \& C-11), $57.7\left(\mathrm{OCH}_{3}\right)$, 66.0 (C-8 \& C-10), 162.9 (C-3), 164.1 (C-5), 200.6 (C=O), 203.1 (C-1).

11c. (0.424 g, 66\%); mp 216-218 ${ }^{\circ} \mathrm{C}$; Anal. Calcd. $\mathrm{C}_{34} \mathrm{H}_{25} \mathrm{C}_{12} \mathrm{~N}_{3} \mathrm{O}_{4} \mathrm{~S}$ : C, 63.55; H, 3.92; N, 6.54. Found: C, 63.50; H, 3.94; N, 6.60. IR (KBr, cm $\left.{ }^{-1}\right) 3260$ \& $3330\left(\mathrm{NH}_{2}\right), 1660$ (COAr), 1680 (CO at 1/1,3); ${ }^{1} \mathrm{H}-\mathrm{NMR}\left(300 \mathrm{MHz}, \mathrm{CDCl}_{3}\right) \delta 1.36(1 \mathrm{H}, \mathrm{SH}, \mathrm{s}), 3.72\left(2 \mathrm{H}, \mathrm{C}_{7} \& \mathrm{C}_{11}-\mathrm{H}, \mathrm{d}, J=9.2 \mathrm{~Hz}\right)$, $5.42\left(2 \mathrm{H}, \mathrm{C}_{8} \& \mathrm{C}_{10}-\mathrm{H}, \mathrm{d}, J=9.2 \mathrm{~Hz}\right), 7.12-7.69(18 \mathrm{H}, \mathrm{Ar}-\mathrm{H}, \mathrm{m}), 9.42\left(2 \mathrm{H}, \mathrm{NH}_{2}, \mathrm{bs}\right) ;{ }^{13} \mathrm{C} \mathrm{NMR}$ $\left(75.5 \mathrm{MHz}, \mathrm{CDCl}_{3}\right) \delta 32.2$ (C-6), 41.5 (C-7 \& C-11), 65.8 (C-8 \& C-10), 163.3 (C-3), 164.6(C5), 201.4(C=O), 203.6(C-1).

12a. (0.360 g, 61\%); mp $243-245^{\circ} \mathrm{C}$; Anal. Calcd. $\mathrm{C}_{34} \mathrm{H}_{27} \mathrm{~N}_{3} \mathrm{O}_{3} \mathrm{~S}_{2}$ : C, 69.25; H, 4.61; N, 7.13. Found: C, 69.15; H, 4.58; N, 7.26. IR (KBr, cm $\left.{ }^{-1}\right) 3250$ \& $3315\left(\mathrm{NH}_{2}\right), 1650$ (COAr), 1665 (CO at 1/1,3); ${ }^{1} \mathrm{H}-\mathrm{NMR}\left(300 \mathrm{MHz}, \mathrm{CDCl}_{3}\right) \delta 1.41(1 \mathrm{H}, \mathrm{SH}, \mathrm{s}), 4.01\left(2 \mathrm{H}, \mathrm{C}_{7} \& \mathrm{C}_{11}-\mathrm{H}, \mathrm{d}, J=9.3 \mathrm{~Hz}\right)$, $4.33\left(2 \mathrm{H}, \mathrm{C}_{8} \& \mathrm{C}_{10}-\mathrm{H}, \mathrm{d}, J=9.3 \mathrm{~Hz}\right), 7.06-7.88(20 \mathrm{H}, \mathrm{Ar}-\mathrm{H}, \mathrm{m}), 9.38\left(2 \mathrm{H}, \mathrm{NH}_{2}\right.$, bs); ${ }^{13} \mathrm{C}-\mathrm{NMR}$ (75.5 MHz, $\mathrm{CDCl}_{3}$ ) $\delta 31.5$ (C-8 \& C-10), 36.4 (C-6), 42.8 (C-7 \& C-11), 164.3 (C-3), 165.4 (C5), 201.2 (C=O), 204.1 (C-1).

12b. (0.448 g, 64\%); mp 188-190 ${ }^{\circ} \mathrm{C}$; Anal. Calcd. $\mathrm{C}_{36} \mathrm{H}_{31} \mathrm{~N}_{3} \mathrm{O}_{5} \mathrm{~S}_{2}$ : C, 66.54; H, 4.81; N, 6.47. Found: C, 66.62; H, 4.85; N, 6.40. IR (KBr, cm $\left.{ }^{-1}\right) 3240$ \& 3305 ( $\left.\mathrm{NH}_{2}\right), 1655$ (COAr), 1670 (CO at 1/1,3); ${ }^{1} \mathrm{H}-\mathrm{NMR}\left(300 \mathrm{MHz}, \mathrm{CDCl}_{3}\right) \delta 1.40(1 \mathrm{H}, \mathrm{SH}, \mathrm{s}), 3.69\left(6 \mathrm{H}, \mathrm{Ar}_{-} \mathrm{OCH}_{3}, \mathrm{~s}\right), 4.04\left(2 \mathrm{H}, \mathrm{C}_{7}\right.$ \& $\left.\mathrm{C}_{11}-\mathrm{H}, \mathrm{d}, J=9.1 \mathrm{~Hz}\right), 4.32\left(2 \mathrm{H}, \mathrm{C}_{8} \& \mathrm{C}_{10}-\mathrm{H}, \mathrm{d}, J=9.1 \mathrm{~Hz}\right), 7.04-7.86(18 \mathrm{H}, \mathrm{Ar}-\mathrm{H}, \mathrm{m}), 9.35$ (2H, $\mathrm{NH}_{2}$, bs); ${ }^{13} \mathrm{C}-\mathrm{NMR}\left(75.5 \mathrm{MHz}, \mathrm{CDCl}_{3}\right) \delta 32.1$ (C-8 \& C-10), 36.7 (C-6), 43.2 (C-7 \& C11), $55.9\left(\mathrm{OCH}_{3}\right), 162.3$ (C-3), 166.4 (C-5), 200.2 (C=O), 202.4 (C-1).

12c. (0.425 g, 69\%); mp 233-235 ${ }^{\circ} \mathrm{C}$; Anal. Calcd. $\mathrm{C}_{34} \mathrm{H}_{25} \mathrm{C}_{12} \mathrm{~N}_{3} \mathrm{O}_{3} \mathrm{~S}_{2}$ : C, 62.00; H, 3.83; N, 6.38. Found: C, 61.95; H, 3.85; N, 6.46. IR (KBr, cm $\left.{ }^{-1}\right) 3242$ \& 3300 (NH2), 1655 (OAr), 1680 (CO at 1/1,3); ${ }^{1} \mathrm{H}-\mathrm{NMR}\left(300 \mathrm{MHz}, \mathrm{CDCl}_{3}\right) \delta 1.38(1 \mathrm{H}, \mathrm{SH}, \mathrm{s}), 4.05\left(2 \mathrm{H}, \mathrm{C}_{7} \& \mathrm{C}_{11}-\mathrm{H}, \mathrm{d}, J=9.0 \mathrm{~Hz}\right)$, $4.34\left(2 \mathrm{H}, \mathrm{C}_{8} \& \mathrm{C}_{10}-\mathrm{H}, \mathrm{d}, J=9.0 \mathrm{~Hz}\right), 7.15-7.91(18 \mathrm{H}, \mathrm{Ar}-\mathrm{H}, \mathrm{m}), 9.33\left(2 \mathrm{H}, \mathrm{NH}_{2}\right.$, bs); ${ }^{13} \mathrm{C}-\mathrm{NMR}$ (75.5 MHz, $\mathrm{CDCl}_{3}$ ) $\delta 33.6$ (C-8 \& C-10), 35.9 (C-6), 41.3 (C-7 \& C-11), 162.0 (C-3), 164.1 (C5), $200.0(\mathrm{C}=\mathrm{O}), 203.5$ (C1).

4-Amino-6,10-diaroyl-7,9-diaryl-2,3,8-triazaspiro[4.5]deca-3-en-1-ones (13) / 4-amino6,10diaroyl-7,9-diaryl-8-oxa-2,3-diazaspiro[4.5]deca-3-en-1-ones (14) / 4-amino-6,10diaroyl-7,9diaryl-8-thia-2,3-diazaspiro[4.5]deca-3-en-1-ones (15). To a solution of $\mathbf{1}$ / 2 / 3 (0.526 / 0.607 / $0.628 \mathrm{~g}, 1 \mathrm{mmol})$ in $\mathrm{MeOH}(20 \mathrm{~mL}), 80 \%$ hydrazine hydrate $(0.075 \mathrm{~g}, 1.5 \mathrm{mmol})$ and 10\% NaOMe $(5 \mathrm{~mL})$ were added and refluxed for $4-5 \mathrm{~h}$. The contents were cooled and poured onto crushed ice containing $\mathrm{HCl}$. The separated solid was filtered, dried and recrystallized from 2propanol.

13a. (0.317 g, 60\%); mp 205-207 ${ }^{\circ}$; Anal. Calcd. $\mathrm{C}_{33} \mathrm{H}_{28} \mathrm{~N}_{4} \mathrm{O}_{3}$ : C, 74.98; H, 5.34; N, 10.60 . Found: C, 74.90; H, 5.28; N, 10.69. IR (KBr, cm $\left.{ }^{-1}\right) 3220$ \& $3280\left(\mathrm{NH}_{2}\right), 3340$ (br, NH), 1640 (COAr), 1660 (CO at 1/1,3); ${ }^{1} \mathrm{H}-\mathrm{NMR}\left(300 \mathrm{MHz}, \mathrm{CDCl}_{3}\right) \delta 3.80\left(2 \mathrm{H}, \mathrm{C}_{6} \& \mathrm{C}_{10}-\mathrm{H}, \mathrm{d}, J=9.1\right.$ $\mathrm{Hz}), 4.58$ (2H, $\left.\mathrm{C}_{7} \& \mathrm{C}_{9}-\mathrm{H}, \mathrm{d}, J=9.1 \mathrm{~Hz}\right), 7.06-7.83$ (20H, Ar-H, m), $9.26\left(4 \mathrm{H}, 2 \mathrm{NH}, \mathrm{NH}_{2}\right.$, bs); 
${ }^{13} \mathrm{C}-\mathrm{NMR}\left(75.5 \mathrm{MHz}, \mathrm{CDCl}_{3}\right) \delta 42.4$ (C-6 \& C-10), 43.9 (C-5), 44.6 (C-7 \& C-9), 155.0 (C-4), $182.1(\mathrm{C}-1), 201.0(\mathrm{C}=\mathrm{O})$.

13b. (0.365 g, 62\%); mp 198-199 ${ }^{\circ} \mathrm{C}$; Anal. Calcd. $\mathrm{C}_{35} \mathrm{H}_{32} \mathrm{~N}_{4} \mathrm{O}_{5}$ : C, 71.41; H, 5.48; N, 9.52. Found: C, 71.52; H, 5.52; N, 9.60. IR $\left(\mathrm{KBr}, \mathrm{cm}^{-1}\right) 3225$ \& $3275\left(\mathrm{NH}_{2}\right), 3345$ (br, NH), 1650 (COAr), 1665 (CO at 1/1,3); ${ }^{1} \mathrm{H}-\mathrm{NMR}\left(300 \mathrm{MHz}, \mathrm{CDCl}_{3}\right) \delta 3.68\left(6 \mathrm{H}, \mathrm{Ar}_{-} \mathrm{OCH}_{3}, \mathrm{~s}\right), 3.82\left(2 \mathrm{H}, \mathrm{C}_{6}\right.$ \& $\left.\mathrm{C}_{10}-\mathrm{H}, \mathrm{d}, J=9.3 \mathrm{~Hz}\right), 4.54\left(2 \mathrm{H}, \mathrm{C}_{7} \& \mathrm{C}_{9}-\mathrm{H}, \mathrm{d}, J=9.3 \mathrm{~Hz}\right), 7.02-7.85$ (18H, Ar-H, m), 9.28 $\left(4 \mathrm{H}, 2 \mathrm{NH}, \mathrm{NH}_{2}\right.$, bs); ${ }^{13} \mathrm{C}-\mathrm{NMR}\left(75.5 \mathrm{MHz}, \mathrm{CDCl}_{3}\right) \delta 43.4$ (C-6 \& C-10), 44.3 (C-5), 46.6 (C-7 \& C-9), $55.9\left(\mathrm{OCH}_{3}\right), 156.0(\mathrm{C}-4), 182.5(\mathrm{C}-1), 205.0(\mathrm{C}=\mathrm{O})$.

13c. (0.388 g, 65\%); mp 215-217 ${ }^{\circ} \mathrm{C}$; Anal. Calcd. $\mathrm{C}_{33} \mathrm{H}_{26} \mathrm{C}_{12} \mathrm{~N}_{4} \mathrm{O}_{3}$ : C, 66.34; H, 4.39; N, 9.38. Found: C, 66.43; H, 4.36; N, 9.44. IR (KBr, cm $\left.{ }^{-1}\right) 3218$ \& $3280\left(\mathrm{NH}_{2}\right), 3343(\mathrm{NH}), 1640$ ( $\underline{\mathrm{CO}} \mathrm{Ar}$ ), 1670 (CO at 1/1,3); ${ }^{1} \mathrm{H}-\mathrm{NMR}\left(300 \mathrm{MHz}, \mathrm{CDCl}_{3}\right) \delta 3.86\left(2 \mathrm{H}, \mathrm{C}_{6} \& \mathrm{C}_{10}-\mathrm{H}, \mathrm{d}, J=9.4\right.$ $\mathrm{Hz}), 4.53\left(2 \mathrm{H}, \mathrm{C}_{7} \& \mathrm{C}_{9}-\mathrm{H}, \mathrm{d}, J=9.4 \mathrm{~Hz}\right), 7.08-7.91(18 \mathrm{H}, \mathrm{Ar}-\mathrm{H}, \mathrm{m}), 9.23\left(4 \mathrm{H}, 2 \mathrm{NH}, \mathrm{NH}_{2}\right.$, bs); ${ }^{13} \mathrm{C}-\mathrm{NMR}\left(75.5 \mathrm{MHz}, \mathrm{CDCl}_{3}\right) \delta 41.5$ (C-6 \& C-10), 44.3 (C-5), 46.2 (C-7 \& C-9), 154.4 (C4), 181.2 (C-1), $202.5(\mathrm{C}=\mathrm{O})$.

14a. (0.339 g, 64\%); mp $188-190^{\circ} \mathrm{C}$; Anal. Calcd. $\mathrm{C}_{33} \mathrm{H}_{27} \mathrm{~N}_{3} \mathrm{O}_{4}$ : C, 74.84; H, 5.14; N, 7.93 . Found: C, 74.92; H, 5.07; N, 7.88. IR (KBr, $\left.\mathrm{cm}^{-1}\right) 3230$ \& $3285\left(\mathrm{NH}_{2}\right), 1645$ (COAr), 1675 (CO at 1/1,3); ${ }^{1} \mathrm{H}-\mathrm{NMR}\left(300 \mathrm{MHz}, \mathrm{CDCl}_{3}\right) \delta 3.92\left(2 \mathrm{H}, \mathrm{C}_{6} \& \mathrm{C}_{10}-\mathrm{H}, \mathrm{d}, J=9.1 \mathrm{~Hz}\right), 5.45\left(2 \mathrm{H}, \mathrm{C}_{7} \&\right.$ $\left.\mathrm{C}_{9}-\mathrm{H}, \mathrm{d}, J=9.1 \mathrm{~Hz}\right), 7.19-7.92\left(20 \mathrm{H}, \mathrm{Ar}-\mathrm{H} \& 3 \mathrm{H}, \mathrm{NH}, \mathrm{NH}_{2}, \mathrm{~m}\right) ;{ }^{13} \mathrm{C}-\mathrm{NMR}\left(75.5 \mathrm{MHz}, \mathrm{CDCl}_{3}\right)$ d41.8 (C-6 \& C-10), 44.0 (C-5), 66.5 (C-7 \& C-9), 153.5 (C-4), 181.8 (C-1), 203.9 (C=O).

14b. (0.389 g, 66\%); mp $177-178^{\circ} \mathrm{C}$; Anal. Calcd. $\mathrm{C}_{35} \mathrm{H}_{31} \mathrm{~N}_{3} \mathrm{O}_{6}$ : C, 71.29; H, 5.30; N, 7.13. Found: C, 71.20; H, 5.33; N, 7.23. IR (KBr, cm $\left.{ }^{-1}\right) 3224$ \& $3292\left(\mathrm{NH}_{2}\right), 1640$ (COAr), 1680 (CO at 1/1,3); ${ }^{1} \mathrm{H}-\mathrm{NMR}\left(300 \mathrm{MHz}, \mathrm{CDCl}_{3}\right) \delta 3.74\left(6 \mathrm{H}, \mathrm{Ar}-\mathrm{OCH}_{3}, \mathrm{~s}\right), 3.95\left(2 \mathrm{H}, \mathrm{C}_{6} \& \mathrm{C}_{10}-\mathrm{H}, \mathrm{d}, J=9.4\right.$ $\mathrm{Hz}), 5.42\left(2 \mathrm{H}, \mathrm{C}_{7} \& \mathrm{C}_{9}-\mathrm{H}, \mathrm{d}, J=9.4 \mathrm{~Hz}\right), 7.09-7.95\left(18 \mathrm{H}, \mathrm{Ar}-\mathrm{H} \& 3 \mathrm{H}, \mathrm{NH}, \mathrm{NH}_{2}, \mathrm{~m}\right) ;{ }^{13} \mathrm{C}$ NMR (75.5 MHz, CDCl $) \delta 42.6$ (C-6 \& C-10), 43.9 (C-5), $55.2\left(\mathrm{OCH}_{3}\right), 64.8$ (C-7 \& C-9), $154.3(\mathrm{C}-$ 4), $183.4(\mathrm{C}-1), 201.5(\mathrm{C}=\mathrm{O})$.

14c. (0.353 g, 59\%); mp 194-196 ${ }^{\circ} \mathrm{C}$; Anal. Calcd. $\mathrm{C}_{33} \mathrm{H}_{25} \mathrm{C}_{12} \mathrm{~N}_{3} \mathrm{O}_{4}$ : C, 66.23; H, 4.21; N, 7.02. Found: C, 66.63; H, 4.25; N, 6.95. IR (KBr, cm $\left.{ }^{-1}\right) 3228$ \& $3290\left(\mathrm{NH}_{2}\right), 1660$ (COAr), 1675 (CO at 1/1,3); ${ }^{1} \mathrm{H}-\mathrm{NMR}\left(300 \mathrm{MHz}, \mathrm{CDCl}_{3}\right) \delta 3.92\left(2 \mathrm{H}, \mathrm{C}_{6} \& \mathrm{C}_{10}-\mathrm{H}, \mathrm{d}, J=9.1 \mathrm{~Hz}\right), 5.40\left(2 \mathrm{H}, \mathrm{C}_{7}\right.$ \& $\left.\mathrm{C}_{9}-\mathrm{H}, \mathrm{d}, J=9.1 \mathrm{~Hz}\right), 7.10-7.96\left(18 \mathrm{H}, \mathrm{Ar}-\mathrm{H} \& 3 \mathrm{H}, \mathrm{NH}, \mathrm{NH}_{2}, \mathrm{~m}\right) ;{ }^{13} \mathrm{C}-\mathrm{NMR}\left(75.5 \mathrm{MHz}, \mathrm{CDCl}_{3}\right)$ d42.4 (C-6 \& C-10), 45.9 (C-5), 63.4 (C-7 \& C-9), 153.6 (C-4), 181.4 (C-1), 202.5 (C=O).

15a. (0.371 g, 68\%); mp 185- $187^{\circ} \mathrm{C}$; Anal. Calcd. $\mathrm{C}_{33} \mathrm{H}_{27} \mathrm{~N}_{3} \mathrm{O}_{3} \mathrm{~S}$ : C, 72.64; H, 4.99; N, 7.70. Found: C, 72.70; H, 5.05; N, 7.78. IR (KBr, $\left.\mathrm{cm}^{-1}\right) 3225$ \& $3295\left(\mathrm{NH}_{2}\right), 1640$ ( $\left.\underline{\mathrm{CO}} \mathrm{Ar}\right), 1668$ (CO at 1/1,3); ${ }^{1} \mathrm{H}-\mathrm{NMR}\left(300 \mathrm{MHz}, \mathrm{CDCl}_{3}\right) \delta 4.03\left(2 \mathrm{H}, \mathrm{C}_{6} \& \mathrm{C}_{10}-\mathrm{H}, \mathrm{d}, J=9.2 \mathrm{~Hz}\right), 4.33\left(2 \mathrm{H}, \mathrm{C}_{7} \&\right.$ $\left.\mathrm{C}_{9}-\mathrm{H}, \mathrm{d}, J=9.2 \mathrm{~Hz}\right), 7.12-7.89\left(2 \mathrm{H}, \mathrm{Ar}-\mathrm{H} \& 3 \mathrm{H}, \mathrm{NH}, \mathrm{NH}_{2}, \mathrm{~m},\right) ;{ }^{13} \mathrm{C}-\mathrm{NMR}\left(75.5 \mathrm{MHz}, \mathrm{CDCl}_{3}\right)$ $\delta 30.6$ (C-7 \& C-9), 42.5 (C-6 \& C-10), 44.1 (C-5), 153.7 (C-4), 180.5 (C-1), 202.3 (C=O).

15b. (0.367 g, 64\%); mp 196-197 ${ }^{\circ} \mathrm{C}$; Anal. Calcd. $\mathrm{C}_{35} \mathrm{H}_{31} \mathrm{~N}_{3} \mathrm{O}_{5} \mathrm{~S}$ : C, 69.40; H, 5.16; N, 6.94. Found: C, 69.47; H, 5.14; N, 7.02. IR (KBr, cm $\left.{ }^{-1}\right) 3230$ \& $3292\left(\mathrm{NH}_{2}\right), 1645$ (COAr), 1676 (CO at 1/1,3); ${ }^{1} \mathrm{H}-\mathrm{NMR}\left(300 \mathrm{MHz}, \mathrm{CDCl}_{3}\right) \delta 3.72\left(6 \mathrm{H}, \mathrm{Ar}-\mathrm{OCH}_{3}, \mathrm{~s}\right), 4.01\left(2 \mathrm{H}, \mathrm{C}_{6} \& \mathrm{C}_{10}-\mathrm{H}, \mathrm{d}, J=9.4\right.$ $\mathrm{Hz}), 4.32\left(2 \mathrm{H}, \mathrm{C}_{7} \& \mathrm{C}_{9}-\mathrm{H}, \mathrm{d}, J=9.4 \mathrm{~Hz}\right), 7.04-7.86\left(18 \mathrm{H}, \mathrm{Ar}-\mathrm{H} \& 3 \mathrm{H}, \mathrm{NH}, \mathrm{NH}_{2}, \mathrm{~m}\right)$; ${ }^{13} \mathrm{C}-\mathrm{NMR}$ 
(75.5 MHz, $\left.\mathrm{CDCl}_{3}\right) \delta 30.2$ (C-7 \& C-9), 41.9 (C-6 \& C-10), 44.5 (C-5), $55.2\left(\mathrm{OCH}_{3}\right), 53.4(\mathrm{C}-4)$, $178.5(\mathrm{C}-1), 204.3(\mathrm{C}=\mathrm{O})$.

15c. (0.411 g, 67\%); mp $169-171^{\circ} \mathrm{C}$; Anal. Calcd. $\mathrm{C}_{33} \mathrm{H}_{25} \mathrm{C}_{22} \mathrm{~N}_{3} \mathrm{O}_{3} \mathrm{~S}$ : C, 64.50; H, 4.10; N, 6.84 . Found: C, 64.45; H, 4.14; N, 7.00. IR (KBr, cm $\left.{ }^{-1}\right) 3230$ \& $3295\left(\mathrm{NH}_{2}\right), 1660$ (COAr), 1680 (CO at 1/1,3); ${ }^{1} \mathrm{H}-\mathrm{NMR}\left(300 \mathrm{MHz}, \mathrm{CDCl}_{3}\right) \delta 4.03\left(2 \mathrm{H}, \mathrm{C}_{6} \& \mathrm{C}_{10}-\mathrm{H}, \mathrm{d}, J=9.0 \mathrm{~Hz}\right), 4.29\left(2 \mathrm{H}, \mathrm{C}_{7} \&\right.$ $\left.\mathrm{C}_{9}-\mathrm{H}, \mathrm{d}, J=9.0 \mathrm{~Hz}\right), 7.09-7.92\left(18 \mathrm{H}, \mathrm{Ar}-\mathrm{H} \& 3 \mathrm{H}, \mathrm{NH}, \mathrm{NH}_{2}, \mathrm{~m}\right) ;{ }^{13} \mathrm{C}-\mathrm{NMR}\left(75.5 \mathrm{MHz}, \mathrm{CDCl}_{3}\right)$ d31.1 (C-7 \& C-9), 42.0 (C-6 \& C-10), 43.7 (C-5), 154.9 (C-4), 182.5 (C-1), 201.8 (C=O).

4-Amino-6,10-diaroyl-7,9-diaryl-2-oxa-3,8-diazaspiro[4.5]deca-3-en-1-ones (16) / 4amino6,10-diaroyl-7,9-diaryl-2,8-dioxa-3-azaspiro[4.5]deca-3-en-1-ones (17) / 4-amino6,10diaroyl-7,9-diaryl-2-oxa-8-thia-3-azaspiro[4.5]deca-3-en-1-ones (18). A mixture of 1 / 2 / 3 (0.526 / 0.607 / 0.628 g, $1 \mathrm{mmol}$ ), hydroxylamine hydrochloride (0.0695 g, $1.5 \mathrm{mmol}$ ), $\mathrm{MeOH}$ $(20 \mathrm{~mL})$ and 10\% NaOMe (5 mL) was refluxed for 4-5 h. It was cooled and poured onto crushed ice containing $\mathrm{HCl}$. The solid obtained was filtered, dried and recrystallized from 2-propanol.

16a. (0.318 g, 60\%); mp 196-198 ${ }^{\circ} \mathrm{C}$; Anal. Calcd. $\mathrm{C}_{33} \mathrm{H}_{27} \mathrm{~N}_{3} \mathrm{O}_{4}$ : C, 74.84; H, 5.14; N, 7.93 . Found: C, 74.97; H, 5.09; N, 8.00. IR (KBr, cm $\left.{ }^{-1}\right) 3214$ \& $3268\left(\mathrm{NH}_{2}\right), 3350(\mathrm{NH}), 1640$ (COAr), 1740 (CO at 1/1,3); ${ }^{1} \mathrm{H}-\mathrm{NMR}\left(300 \mathrm{MHz}, \mathrm{CDCl}_{3}\right) \delta 3.82\left(2 \mathrm{H}, \mathrm{C}_{6} \& \mathrm{C}_{10}-\mathrm{H}, \mathrm{d}, J=9.0\right.$ $\mathrm{Hz}$ ), 4.53 (2H, C 7 \& $\mathrm{C}_{9}-\mathrm{H}, \mathrm{d}, J=9.0 \mathrm{~Hz}$ ), 7.12-7.79 (20H, Ar-H, m), 9.81 (3H, NH, NH 2 , bs); ${ }^{13} \mathrm{C}-\mathrm{NMR}\left(75.5 \mathrm{MHz}, \mathrm{CDCl}_{3}\right.$ ) $\delta 36.6$ (C-6 \& C-10), 44.7 (C-7 \& C-9), 49.5 (C-5), 163.4 (C-4), $181.0(\mathrm{C}-1), 203.2(\mathrm{C}=\mathrm{O})$.

16b. (0.336 g, 57\%); mp 198-200 ${ }^{\circ} \mathrm{C}$; Anal. Calcd. $\mathrm{C}_{35} \mathrm{H}_{31} \mathrm{~N}_{3} \mathrm{O}_{6}$ : C, 71.29; H, 5.30; N, 7.13. Found: C, 71.21; H, 5.36; N, 7.20. IR (KBr, $\left.\mathrm{cm}^{-1}\right) 3222$ \& $3275\left(\mathrm{NH}_{2}\right), 3347(\mathrm{NH}), 1645$ (COAr), 1745 (CO at 1/1,3); ${ }^{1} \mathrm{H}-\mathrm{NMR}\left(300 \mathrm{MHz}, \mathrm{CDCl}_{3}\right) \delta 3.69\left(6 \mathrm{H}, \mathrm{Ar}_{-} \mathrm{OCH}_{3}, \mathrm{~s}\right), 3.83\left(2 \mathrm{H}, \mathrm{C}_{6}\right.$ \& $\left.\mathrm{C}_{10}-\mathrm{H}, \mathrm{d}, J=9.3 \mathrm{~Hz}\right), 4.52\left(2 \mathrm{H}, \mathrm{C}_{7} \& \mathrm{C}_{9}-\mathrm{H}, \mathrm{d}, J=9.3 \mathrm{~Hz}\right), 7.08-7.79$ (18H, Ar-H, m), 9.87 (3H, NH, $\mathrm{NH}_{2}$, bs); ${ }^{13} \mathrm{C}-\mathrm{NMR}\left(75.5 \mathrm{MHz}, \mathrm{CDCl}_{3}\right) \delta 37.8$ (C-6 \& C-10), 45.0 (C-7 \& C-9), 48.2 (C-5), $55.8\left(\mathrm{OCH}_{3}\right), 162.1(\mathrm{C}-4), 182.6(\mathrm{C}-1), 201.6(\mathrm{C}=\mathrm{O})$.

16c. (0.395 g, 66\%); mp 175-176 ${ }^{\circ} \mathrm{C}$; Anal. Calcd. $\mathrm{C}_{33} \mathrm{H}_{25} \mathrm{C}_{12} \mathrm{~N}_{3} \mathrm{O}_{4}$ : C, 66.23; H, 4.21; N, 7.02. Found: C, 66.33; H, 4.18; N, 7.08. IR (KBr, cm $\left.{ }^{-1}\right) 3218$ \& $3272\left(\mathrm{NH}_{2}\right), 3352(\mathrm{NH}), 1660$ ( $\underline{\mathrm{CO}} \mathrm{Ar}$ ), 1750 (CO at 1/1,3); ${ }^{1} \mathrm{H}-\mathrm{NMR}\left(300 \mathrm{MHz}, \mathrm{CDCl}_{3}\right) \delta 3.87\left(2 \mathrm{H}, \mathrm{C}_{6} \& \mathrm{C}_{10}-\mathrm{H}, \mathrm{d}, J=9.4\right.$ $\mathrm{Hz}), 4.55$ (2H, C 7 \& $\mathrm{C}_{9}-\mathrm{H}, \mathrm{d}, J=9.4 \mathrm{~Hz}$ ), 7.16-7.81 (18H, Ar-H, m), 9.88 (3H, NH, NH 2 , bs); ${ }^{13} \mathrm{C}-\mathrm{NMR}\left(75.5 \mathrm{MHz}, \mathrm{CDCl}_{3}\right) \delta 37.4$ (C-6 \& C-10), 44.5 (C-7 \& C-9), 47.8 (C-5), 163.2 (C4), 181.7 (C-1), 200.5 (C=O).

17a. (0.355 g, 65\%); mp 205-206 ${ }^{\circ} \mathrm{C}$; Anal. Calcd. $\mathrm{C}_{33} \mathrm{H}_{26} \mathrm{~N}_{2} \mathrm{O}_{5}$ : C, 74.70; H, 4.94; N, 5.28. Found: C, 74.60; H, 4.90; N, 5.22. IR (KBr, $\left.\mathrm{cm}^{-1}\right) 3235$ \& $3300\left(\mathrm{NH}_{2}\right), 1650$ ( $\left.\underline{\mathrm{COAr}}\right), 1744$ (CO at 1/1,3); ${ }^{1} \mathrm{H}-\mathrm{NMR}\left(300 \mathrm{MHz}, \mathrm{CDCl}_{3}\right) \delta 4.27\left(2 \mathrm{H}, \mathrm{C}_{6} \& \mathrm{C}_{10}-\mathrm{H}, \mathrm{d}, J=9.2 \mathrm{~Hz}\right), 5.36\left(2 \mathrm{H}, \mathrm{C}_{7} \&\right.$ $\left.\mathrm{C}_{9}-\mathrm{H}, \mathrm{d}, J=9.2 \mathrm{~Hz}\right), 7.19-7.83(20 \mathrm{H}, \mathrm{Ar}-\mathrm{H}, \mathrm{m}), 9.28\left(2 \mathrm{H}, \mathrm{NH}_{2}, \mathrm{bs}\right) ;{ }^{13} \mathrm{C}-\mathrm{NMR}(75.5 \mathrm{MHz}$, $\left.\mathrm{CDCl}_{3}\right) \delta 36.6$ (C-6 \& C-10), 48.4 (C-5), 65.2 (C-7 \& C-9), 162.9 (C-4), 179.6 (C-1), 201.6 $(\mathrm{C}=\mathrm{O})$.

17b. (0.402 g, 68\%); mp 221-223 ${ }^{\circ} \mathrm{C}$; Anal. Calcd. $\mathrm{C}_{35} \mathrm{H}_{30} \mathrm{~N}_{2} \mathrm{O}_{7}$ : C, 71.18; H, 5.12; N, 4.74. Found: C, 71.26; H, 5.10; N, 4.80. IR (KBr, $\left.\mathrm{cm}^{-1}\right) 3232$ \& $3298\left(\mathrm{NH}_{2}\right), 1640$ (을), 1748 (CO 
at 1/1,3); ${ }^{1} \mathrm{H}-\mathrm{NMR}\left(300 \mathrm{MHz}, \mathrm{CDCl}_{3}\right) \delta 3.68\left(6 \mathrm{H}, \mathrm{Ar}_{-} \mathrm{OCH}_{3}, \mathrm{~s}\right), 4.25\left(2 \mathrm{H}, \mathrm{C}_{6} \& \mathrm{C}_{10}-\mathrm{H}, \mathrm{d}, J=9.1\right.$ $\mathrm{Hz}), 5.37$ (2H, C7 \& $\left.\mathrm{C}_{9}-\mathrm{H}, \mathrm{d}, J=9.1 \mathrm{~Hz}\right), 7.03-7.80(18 \mathrm{H}, \mathrm{Ar}-\mathrm{H}, \mathrm{m}), 9.25\left(2 \mathrm{H}, \mathrm{NH}_{2}\right.$, bs); ${ }^{13} \mathrm{C}-$ NMR (75.5 MHz, CDCl 3 ) $\delta 37.5\left(\mathrm{C}-6\right.$ \& C-10), $47.8(\mathrm{C}-5), 55.8\left(\mathrm{OCH}_{3}\right), 65.4$ (C-7 \& C-9), $163.2(\mathrm{C}-4), 178.6(\mathrm{C}-1), 202.2(\mathrm{C}=\mathrm{O})$.

17c. (0.372 g, 62\%); mp 214-216 ${ }^{\circ} \mathrm{C}$; Anal. Calcd. $\mathrm{C}_{33} \mathrm{H}_{24} \mathrm{C}_{12} \mathrm{~N}_{2} \mathrm{O}_{5}$ : C, 66.12; H, 4.04; N, 4.67. Found: C, 66.20; H, 4.08; N, 4.72. IR (KBr, cm $\left.{ }^{-1}\right) 3235$ \& $3302\left(\mathrm{NH}_{2}\right), 1655$ (COAr), 1750 (CO at 1/1,3); ${ }^{1} \mathrm{H}-\mathrm{NMR}\left(300 \mathrm{MHz}, \mathrm{CDCl}_{3}\right) \delta 4.34\left(\mathrm{~d}, 2 \mathrm{H}, \mathrm{C}_{6} \& \mathrm{C}_{10}-\mathrm{H}, J=9.2 \mathrm{~Hz}\right), 5.42\left(\mathrm{~d}, 2 \mathrm{H}, \mathrm{C}_{7} \&\right.$ $\mathrm{C}_{9}-\mathrm{H}, J=9.2 \mathrm{~Hz}$ ), 7.13-7.79 (m, 18H, Ar-H), 9.32 (bs, $\left.2 \mathrm{H}, \mathrm{NH}_{2}\right) ;{ }^{13} \mathrm{C}-\mathrm{NMR}\left(75.5 \mathrm{MHz}, \mathrm{CDCl}_{3}\right)$ $\delta 37.9$ (C-6 \& C-10), 48.4 (C-5), 66.4 (C-7 \& C-9), 162.9 (C-4), 179.6 (C-1), 201.6 (C=O).

18a. (0.328 g, 60\%); mp 182-184 ${ }^{\circ} \mathrm{C}$; Anal. Calcd. $\mathrm{C}_{33} \mathrm{H}_{26} \mathrm{~N}_{2} \mathrm{O}_{4} \mathrm{~S}$ : C, 72.51; H, 4.79; N, 5.12. Found: C, 72.64; H, 4.83; N, 5.24. IR (KBr, cm $\left.{ }^{-1}\right) 3230$ \& $3304\left(\mathrm{NH}_{2}\right), 1645$ (COAr), 1746 (CO at 1/1,3); ${ }^{1} \mathrm{H}-\mathrm{NMR}\left(300 \mathrm{MHz}, \mathrm{CDCl}_{3}\right) \delta 4.04\left(2 \mathrm{H}, \mathrm{C}_{6} \& \mathrm{C}_{10} \mathrm{-H}, \mathrm{d}, J=9.4 \mathrm{~Hz}\right), 4.33\left(2 \mathrm{H}, \mathrm{C}_{7} \&\right.$ $\left.\mathrm{C}_{9}-\mathrm{H}, \mathrm{d}, J=9.4 \mathrm{~Hz}\right), 7.08-7.87(20 \mathrm{H}, \mathrm{Ar}-\mathrm{H}, \mathrm{m}), 9.29\left(2 \mathrm{H}, \mathrm{NH}_{2}, \mathrm{bs}\right) ;{ }^{13} \mathrm{C}-\mathrm{NMR}(75.5 \mathrm{MHz}$, $\mathrm{CDCl}_{3}$ ) $\delta 31.3$ (C-7 \& C-9), 36.5 (C-6 \& C-10), 49.1 (C-5), 162.9 (C-4), 179.6 (C-1), 203.8 $(\mathrm{C}=\mathrm{O})$.

18b. (0.376 g, 62\%); mp 196-197 ${ }^{\circ} \mathrm{C}$; Anal. Calcd. $\mathrm{C}_{35} \mathrm{H}_{30} \mathrm{~N}_{2} \mathrm{O}_{6} \mathrm{~S}$ : C, 69.29; H, 4.98; N, 4.62. Found: C, 69.30; H, 4.95; N, 4.68. IR (KBr, cm $\left.{ }^{-1}\right) 3231$ \& $3300\left(\mathrm{NH}_{2}\right), 1650$ (COAr), 1742 (CO at 1/1,3); ${ }^{1} \mathrm{H}-\mathrm{NMR}\left(300 \mathrm{MHz}, \mathrm{CDCl}_{3}\right) \delta 3.78\left(6 \mathrm{H}, \mathrm{Ar}-\mathrm{OCH}_{3}, \mathrm{~s}\right), 4.04\left(2 \mathrm{H}, \mathrm{C}_{6} \& \mathrm{C}_{10}-\mathrm{H}, \mathrm{d}, J=9.2\right.$ $\mathrm{Hz}), 4.35\left(2 \mathrm{H}, \mathrm{C}_{7} \& \mathrm{C}_{9}-\mathrm{H}, \mathrm{d}, J=9.2 \mathrm{~Hz}\right), 7.06-7.79(18 \mathrm{H}, \mathrm{Ar}-\mathrm{H}, \mathrm{m}), 9.32\left(2 \mathrm{H}, \mathrm{NH}_{2}, \mathrm{bs}\right) ;{ }^{13} \mathrm{C}$ NMR (75.5 MHz, CDCl 3 ) $\delta 32.1$ (C-7 \& C-9), 37.2 (C-6 \& C-10), $50.4(\mathrm{C}-5), 56.8\left(\mathrm{OCH}_{3}\right)$, 163.3 (C-4), $180.2(\mathrm{C}-1), 201.5(\mathrm{C}=\mathrm{O})$.

18c. (0.400 g, 65\%); mp $168-170^{\circ} \mathrm{C}$; Anal. Calcd. $\mathrm{C}_{33} \mathrm{H}_{24} \mathrm{C}_{12} \mathrm{~N}_{2} \mathrm{O}_{4} \mathrm{~S}$ : C, 64.39; H, 3.93; N, 4.55. Found: C, 64.32; H, 3.97; N, 4.60. IR (KBr, cm $\left.{ }^{-1}\right) 3236$ \& 3307 ( $\left.\mathrm{NH}_{2}\right), 1660$ (COAr), 1745 (CO at 1/1,3); ${ }^{1} \mathrm{H}-\mathrm{NMR}\left(300 \mathrm{MHz}, \mathrm{CDCl}_{3}\right) \delta 4.06\left(2 \mathrm{H}, \mathrm{C}_{6} \& \mathrm{C}_{10} \mathrm{-H}, \mathrm{d}, J=9.1 \mathrm{~Hz}\right), 4.38\left(2 \mathrm{H}, \mathrm{C}_{7} \&\right.$ $\left.\mathrm{C}_{9}-\mathrm{H}, \mathrm{d}, J=9.1 \mathrm{~Hz}\right), 7.16-7.81(18 \mathrm{H}, \mathrm{Ar}-\mathrm{H}, \mathrm{m}), 9.30\left(2 \mathrm{H}, \mathrm{NH}_{2}\right.$, bs); ${ }^{13} \mathrm{C}-\mathrm{NMR}(75.5 \mathrm{MHz}$, $\mathrm{CDCl}_{3}$ ) $\delta 32.4$ (C-7 \& C-9), 38.3 (C-6 \& C-10), 50.8 (C-5), 164.8 (C-4), 182.7 (C-1), 202.5 $(\mathrm{C}=\mathrm{O})$.

\section{Acknowledgements}

The authors are thankful to CSIR, New Delhi, India for the financial assistance under major research project.

\section{References}

1. Bhaskar Reddy, D.; Padmavathi, V.; Ramana Reddy, P. V. Indian J. Chem. 1992, 31B, 774.

2. Bhaskar Reddy, D.; Padmavathi, V.; Seenaiah, B.; Padmaja, A. Heteroatom Chem. 1993, 4, 55. 
3. Bhaskar Reddy, D.; Ramana Reddy, M.V.; Padmavathi, V. Indian J. Chem. 1998, 37B, 167.

4. Bhaskar Reddy, D.; Ramana Reddy, M. V.; Padmavathi, V. Heteroatom Chem. 1999, 10, 17.

5. Bhaskar Reddy, D.; Chandrasekhar Babu, N.; Padmavathi, V. Heteroatom Chem. 2001, 12, 131.

6. Bhaskar Reddy, D.; Chandrasekhar Babu, N.; Padmavathi, V. J. Heterocycl. Chem. 2001, 38, 769.

7. Padmavathi, V.; Balaiah, A.; Venugopal Reddy, K.; Padmaja, A.; Bhaskar Reddy, D. Indian J. Chem. 2002, 41B, 1670.

8. Padmavathi, V.; Balaiah, A.; Padmaja, A.; Bhaskar Reddy, D. Phosphorus, Sulfur and Silicon 2002, 177, 2791.

9. Padmavathi, V.; Jagan Mohan Reddy, B.; Rajagopala Sarma, M.; Padmaja, A. Indian J. Chem. 2004, 43B, 2459.

10. 10. Padmavathi, V.; Balaiah, A.; Ramana Reddy, T. V.; Jagan Mohan Reddy, B.; Bhaskar Reddy, D. Heteroatom Chem. 2003, 14, 513.

11. Padmavathi, V.; Balaiah, A.; Bhaskar Reddy, D. J. Heterocycl. Chem. 2002, 39, 649.

12. Padmavathi, V.; Balaiah, A.; Muralidhar Reddy, M.; Bhaskar Reddy, D. Indian J. Chem. 2003, 42B, 1519.

13. Padmavathi, V.; Jagan Mohan Reddy, B.; Padmaja, A.; Venugopal Reddy, K.; Rajagopala Sarma, M.; Indian J. Chem. 2004, 43B, 2628.

14. Bhaskar Reddy, D.; Somasekhar Reddy, A.; Padmavathi, V. Indian J. Chem. 1999, 38, 141.

15. Vincent, J. C.; Vincent, H. W.; Proc. Soc. Expt. Biol. Med. 1944, 55, 162. 